NBER WORKING PAPER SERIES

\title{
HIGH- AND LOW-FREQUENCY EXCHANGE RATE VOLATILITY DYNAMICS: RANGE-BASED ESTIMATION OF STOCHASTIC VOLATILITY MODELS
}

\author{
Sassan Alizadeh \\ Michael W. Brandt \\ Francis X. Diebold \\ Working Paper 8162 \\ http://www.nber.org/papers/w8162 \\ NATIONAL BUREAU OF ECONOMIC RESEARCH \\ 1050 Massachusetts Avenue \\ Cambridge, MA 02138 \\ March 2001
}

This work was supported by the National Science Foundation and the Rodney L. White Center for Financial Research at the Wharton School. Siem Koopman graciously shared both his wisdom and his Ox routines. We thank the editor and referee for their extensive and insightful suggestions. We are also grateful to Torben Andersen, Tim Bollerslev, Steve Brown, Freddy Delbaen, Rob Engle, Eric Ghysels, Joel Hasbrouck, Chris Jones, Ken Kavajecz, Leonid Kogan, Andrew Patton, Chris Rogers, Steve Satchell, George Tauchen, and seminar and conference participants at Carnegie Mellon University, Columbia University, Goldman Sachs Asset Management, the University of Pennsylvania, the University of Rochester, the June 2000 meeting of the Western Finance Association, and the July 2000 NBER Summer Institute. Paul Labys, Canlin Li, Dmitry Livdan, and Clara Vega provided able research assistance. Parts of this paper were written while Diebold visited the Stern School of Business, New York University, whose hospitality is gratefully acknowledged. The views expressed herein are those of the authors and not necessarily those of the National Bureau of Economic Research.

(C) 2001 by Sassan Alizadeh, Michael W. Brandt and Francis X. Diebold. All rights reserved. Short sections of text, not to exceed two paragraphs, may be quoted without explicit permission provided that full credit, including $(\mathrm{C}$ notice, is given to the source. 
High- and Low-Frequency Exchange Rate Volatility Dynamics:

Range-Based Estimation of Stochastic Volatility Models

Sassan Alizadeh, Michael W. Brandt and Francis X. Diebold

NBER Working Paper No. 8162

March 2001

JEL No.G1, F3, C1

\begin{abstract}
We propose using the price range in the estimation of stochastic volatility models. We show theoretically, numerically, and empirically that the range is not only a highly efficient volatility proxy, but also that it is approximately Gaussian and robust to microstructure noise. The good properties of the range imply that range-based Gaussian quasi-maximum likelihood estimation produces simple and highly efficient estimates of stochastic volatility models and extractions of latent volatility series. We use our method to examine the dynamics of daily exchange rate volatility and discover that traditional one-factor models are inadequate for describing simultaneously the high- and low-frequency dynamics of volatility. Instead, the evidence points strongly toward twofactor models with one highly persistent factor and one quickly mean-reverting factor.
\end{abstract}

Sassan Alizadeh

Parametric Capital Management

Francis X. Diebold

University of Pennsylvania

and NBER
Michael W. Brandt

University of Pennsylvania

and NBER 
Volatility is a central concept in finance, whether in asset pricing, portfolio choice, or risk management. Not long ago, theoretical models routinely assumed constant volatility (e.g., Merton, 1969; Black and Scholes, 1973). Today, however, we widely acknowledge that volatility is both time-varying and predictable (e.g., Andersen and Bollerslev, 1997), and stochastic volatility models are commonplace. Discrete- and continuous-time stochastic volatility models are extensively used in theoretical finance, empirical finance, and financial econometrics, both in academe and industry (e.g., Hull and White, 1987; Heston, 1993; Bates, 1996; Ghysels, Harvey, and Renault, 1996; Jarrow, 1998; Duffie, Pan, and Singleton, 2000). Unfortunately, the estimation of stochastic volatility models has proved quite difficult. The Gaussian quasi-maximum likelihood estimation (QMLE) approach of Ruiz (1994) and Harvey, Ruiz, and Shephard (1994), which initially seemed appealing because of its simplicity, fell by the wayside as it became apparent that stochastic volatility models are highly nonGaussian. The problem is that standard volatility proxies such as log absolute or squared returns are contaminated by highly non-Gaussian measurement error (e.g., Andersen and Sorensen, 1997), which produces highly inefficient Gaussian quasi-maximum likelihood estimators and similarly inefficient inferences about latent volatility.

The literature therefore turned toward alternative estimators. In particular, attention turned to variants of the generalized method of moments (GMM) that use model moments obtained either through simulations (e.g., Duffie and Singleton, 1993) or analytically (e.g., Singleton, 1997). Those estimators, however, can also be highly inefficient, depending on the choice of moment conditions and weighting matrix. Although recent GMM work has tried to maximize efficiency through the optimal choice of moment conditions, empirical implementation 
remains challenging (e.g., Gallant, Hsieh, and Tauchen, 1997; Gallant, Hsu, and Tauchen, 1999; Chernov and Ghysels, 2000).

Another literature focuses on likelihood-based estimation and evaluates the likelihood function either through numerical integration (e.g., Fridman and Harris, 1998) or Monte Carlo integration using either importance sampling (e.g., Danielsson, 1994; Sandmann and Koopman, 1998) or Markov Chain methods (e.g., Jacquier, Polson, and Rossi, 1994; Kim, Shephard, and Chib, 1998). In principle, both numerical and Monte Carlo integration can deliver highly accurate approximations to the exact maximum likelihood estimator, but practical considerations have impeded their widespread use. In particular, the methods are computationally intensive and rely on assumptions that are hard to check in practice, such as the accuracy of numerical integrals and the convergence of simulated Markov chains to their steady state.

Motivated both by the popularity and appeal of stochastic volatility models and by the difficulties associated with estimating them, we propose a simple yet highly efficient estimation method based on the range. The range, defined as the difference between the highest and lowest $\log$ security price over a fixed sampling interval, is a volatility proxy with a long and colorful history in finance (e.g., Garman and Klass, 1980; Parkinson, 1980; Beckers, 1983; Ball and Torous 1984; Rogers and Satchell, 1991; Anderson and Bollerslev, 1998; Yang and Zhang, 2000). Data on the range are widely available for individual stocks and exchange-traded futures contracts (including stock indices, Treasury securities, commodities, and currencies), not only at present but also over long historical spans. In fact, the range has been reported for many years in major business newspapers through so-called "candlestick plots," showing the daily high, low, 
and close. The range is also a popular technical indicator (e.g., Edwards and Magee, 1997).

Curiously, however, the range has been neglected in the recent stochastic volatility literature. ${ }^{1}$

The methodological contribution of the paper unfolds in Sections I through III. We set the stage for the paper in Section I, in which we describe a general class of continuous-time stochastic volatility models and the particular discretization that we exploit. In Section II we use both analytical and numerical methods to motivate and establish the remarkable near-normality of the log range. We also note that the log range is a highly efficient volatility measure, a fact known at least since Parkinson (1980) and recently formalized by Andersen and Bollerslev (1998). The approximate normality and high efficiency of the log range suggest its use in Gaussian quasi-maximum likelihood estimation. We pursue this idea in the Monte Carlo study of Section III, which reveals not only huge efficiency gains from our approach relative to traditional methods, but also robustness to microstructure noise.

In Section IV we use the new range-based methods to perform a detailed empirical analysis of volatility dynamics in five major U.S. dollar exchange rates, which delivers sharp new insights. In particular, we find that two-factor models are clearly required to explain both the autocorrelation of volatility and the volatility of volatility, a result that is consistent with both economic theories and empirical studies of volatility dynamics in other markets. Finally, in Section V we summarize, conclude, and sketch directions for future research.

\footnotetext{
${ }^{1}$ Schwert (1990) and Gallant, Hsu, and Tauchen (1999) also make use of the range, albeit with a very different estimator. Although they are aware of the efficiency of the range as a volatility measure, they are unaware of and do not exploit its log-normality, just as in the earlier Garman-Klass-Parkinson literature.
} 


\section{Stochastic Volatility}

\section{A. Continuous-Time Stochastic Volatility Model}

In a generic continuous-time stochastic volatility model, the price $S$ of a security evolves as a diffusion with instantaneous drift $\mu$ and volatility $\sigma$. Both the drift and volatility depend on a latent state variable $v$, which itself evolves as a diffusion. Formally, we write:

$$
\begin{aligned}
& d S_{t}=\mu\left(S_{t}, v_{t}\right) d t+\sigma\left(S_{t}, v_{t}\right) d W_{S t} \\
& d v_{t}=\alpha\left(S_{t}, v_{t}\right) d t+\beta\left(S_{t}, v_{t}\right) d W_{v t},
\end{aligned}
$$

where $W_{S t}$ and $W_{v t}$ are two Wiener processes with correlation $d W_{S t} d W_{v t}=\theta\left(S_{t}, v_{t}\right) d t$. The functions $\alpha$ and $\beta$ govern the drift and volatility of the state variable process.

The stochastic volatility literature contains numerous variations on the generic model (1). In this paper we work with a first-order parameterization, which is rich enough to be interesting, yet simple enough to permit a streamlined exposition:

$$
\begin{gathered}
\frac{d S_{t}}{S_{t}}=\mu d t+\sigma_{t} d W_{S t} \\
d \ln \sigma_{t}=\alpha\left(\ln \bar{\sigma}-\ln \sigma_{t}\right) d t+\beta d W_{v t}
\end{gathered}
$$

The simple stochastic volatility model (2) emerges from the general model (1) when $\sigma\left(S_{t}, v_{t}\right)=\sigma_{t} S_{t}, \sigma_{t}=\exp \left(v_{t}\right), \alpha\left(S_{t}, v_{t}\right)=\alpha\left(\ln \bar{\sigma}-v_{t}\right), \beta\left(S_{t}, v_{t}\right)=\beta$, and $\theta\left(S_{t}, v_{t}\right)=0$. In this parameterization, the $\log$ volatility $\ln \sigma$ of returns $d S / S$ is the latent state variable. It evolves as a mean-reverting Ornstein-Uhlenbeck process, with mean $\ln \bar{\sigma}$ and mean reversion parameter $\alpha>0$. The instantaneous drift of returns and the instantaneous drift and standard deviation of log 
volatility are assumed constant, and the return innovations are assumed independent of the log volatility innovations. $^{2}$

\section{B. Discretization of the Continuous-Time Model}

In practice, we have to rely on $N$ discrete-time price realizations to draw inference about the continuous-time model. Thus, we divide the sample period $[0, T]$ into $N$ intervals, each of length $H=T / N$, corresponding to the discrete-time data. ${ }^{3}$ We then replace the continuous volatility dynamics with a piecewise-constant process, where within each interval $i$, that is between times $i H$ and $(i+1) H$, for $i=1,2, \ldots, N$, volatility is assumed constant at $\sigma_{t}=\sigma_{i H}$, but from one interval to the next, volatility is stochastic.

This piecewise-constant approximation implies that within each interval $i$ the security price evolves as a geometric Brownian motion:

$$
\frac{d S_{t}}{S_{t}}=\mu d t+\sigma_{i H} d W_{S t}, \quad \text { for } i H<t \leq(i+1) H
$$

and, by Ito's lemma, that the $\log$ security price $s_{t}=\ln S_{t}$ evolves as a Brownian motion:

$$
d s_{t}=\left(\mu-\frac{1}{2} \sigma_{i H}^{2}\right) d t+\sigma_{i H} d W_{S t}, \quad \text { for } i H<t \leq(i+1) H .
$$

\footnotetext{
${ }^{2}$ We maintain the zero correlation assumption for tractability. It rules out the leverage effect in volatilities (e.g., Schwert, 1989; Nelson, 1991; Engle and Ng, 1993; Jacquier, Polson, and Rossi, 1999). Our estimator can, however, be extended to allow for non-zero correlation along the lines of Harvey and Shephard (1996); see Alizadeh (1998).

${ }^{3}$ The assumption of equally-spaced observations is made for notational convenience and can be relaxed.
} 
Log volatility varies from one interval to the next according to its Ornstein-Uhlenbeck dynamics. For small interval lengths $H$, the conditional distribution of log volatility is approximately: ${ }^{4,5}$

$$
\ln \sigma_{(i+1) H} \mid \ln \sigma_{i H} \sim \mathrm{N}\left[\ln \bar{\sigma}+\rho_{H}\left(\ln \sigma_{i H}-\ln \bar{\sigma}\right), \beta^{2} H\right] .
$$

In words, the discretized log volatility follows a Gaussian first-order autoregressive process with mean $\ln \bar{\sigma}$, autoregressive parameter $\rho_{H}=1-\alpha H$, and variance $\beta^{2} H$.

\section{Econometric Approach}

\section{A. Measuring Volatility}

Even the discretized stochastic volatility model is difficult to estimate because the sample path of the asset price within each interval is not fully observed. If it were observed, we could infer the diffusion coefficients $\sigma_{i H}$ with arbitrary precision. ${ }^{3}$ In practice, we are forced to use discretely observed statistics of the sample paths, such as the absolute or squared returns over each interval, to draw inferences about the discretized log volatilities and their dynamics.

To formalize this idea, consider a volatility proxy that is a statistic $f\left(s_{i H,(i+1) H}\right)$ of the continuous sample path $s_{i H,(i+1) H}$ of the log asset price between times $i H$ and $(i+1) H$. If the statistic is homogeneous in some power $\gamma$ of volatility, then we can write it as:

$$
f\left(s_{i H,(i+1) H}\right)=\sigma_{i H}^{\gamma} f\left(s_{i H,(i+1) H}^{*}\right),
$$

\footnotetext{
${ }^{4}$ This conditional distribution is an approximation for small $H$. The exact conditional distribution of $\ln \sigma_{(i+1) H}$ is normal with mean $\ln \bar{\sigma}+\exp (-\alpha H)\left(\ln \sigma_{i H}-\ln \bar{\sigma}\right)$ and variance $\beta^{2}[1-\exp (-2 \alpha H)] /(2 \alpha)$. The approximation follows from Taylor series expansions of $\exp (-\alpha H)$ and $\exp (-2 \alpha H)$ around $H=0$.

${ }^{5}$ A number of stochastic volatility papers postulate the discretized volatility dynamics (5) from the onset (e.g., Jacquier, Polson, and Rossi, 1999). We could do the same without loss of generality, except that we need the continuous-time price dynamics (3) and (4) to derive the properties of the volatility proxies in Section II.

${ }^{3}$ See, for example, Merton (1980).
} 
which implies that:

$$
\ln \left|f\left(s_{i H,(i+1) H}\right)\right|=\gamma \ln \sigma_{i H}+\ln \left|f\left(s_{i H,(i+1) H}^{*}\right)\right|,
$$

where $s_{i H,(i+1) H}^{*}$ denotes the continuous sample path of a standardized diffusion generated by the same innovations as $s_{i H,(i+1) H}$, but with volatility $\sigma_{i H}^{*}=1$.

Equation (7) makes clear that the statistic $f(\cdot)$ is a noisy volatility proxy: the first term is proportional to log volatility and the second term is a measurement error. Other things the same, the measurement error reduces the informational content of the volatility proxy. The more variable the measurement error, the less precise are our inferences about log volatility and its dynamics.

\section{B. Linear State Space Representation}

Following Ruiz (1994) and Harvey, Ruiz, and Shephard (1994), we recognize that equations (5) and (7) form a linear state space system:

$$
\begin{gathered}
\ln \sigma_{(i+1) H}=\ln \bar{\sigma}+\rho_{H}\left(\ln \sigma_{i H}-\ln \bar{\sigma}\right)+\beta \sqrt{H} v_{(i+1) H} \\
\ln \left|f\left(s_{i H,(i+1) H}\right)\right|=\gamma \ln \sigma_{i H}+\mathrm{E}\left[\left|f\left(s_{i H,(i+1) H}^{*}\right)\right|\right]+\varepsilon_{(i+1) H}
\end{gathered}
$$

The transition equation (8a) follows from the conditional distribution of log volatility. It describes the dynamics of the unobserved log volatility. The transition errors $v$ are i.i.d. N[0,1], which follows from equation (5). The measurement equation (8b) makes precise the way in which the $\log$ volatility proxy $\ln |f(\cdot)|$ is related to the true $\log$ volatility $\ln \sigma_{i H}$; it follows from equation (7) with the projection $\ln |f(\cdot)| \equiv \mathrm{E}[\ln |f(\cdot)|]+\varepsilon$. The expectation of $\left|f\left(s_{i H,(i+1) H}^{*}\right)\right|$ depends on $s_{i H}^{*}$, the functional form of $f(\cdot)$, and interval length $H$, but it is by construction 
independent of the $\log$ volatility $\ln \sigma_{i H}$. The projection errors $\varepsilon$ have a zero mean but are not necessarily Gaussian.

\section{Quasi-Maximum Likelihood Estimation}

If the measurement equation errors are Gaussian, exact maximum likelihood estimation of the stochastic volatility model is straightforward. One simply maximizes the Gaussian log likelihood:

$$
\ln \mathscr{L}\left(\ln \left|f\left(s_{0, H}\right)\right|, \ln \left|f\left(s_{H, 2 H}\right)\right|, \ldots, \ln \left|f\left(s_{(N-1) H, N H}\right)\right| ; \theta\right)=c-\frac{1}{2} \sum_{i=1}^{N} \ln \eta_{i}-\frac{1}{2} \sum_{i=1}^{N} \frac{e_{i}^{2}}{\eta_{i}},
$$

where the one-step ahead forecast errors:

$$
e_{i}=\ln \left|f\left(s_{(i-1) H, i H}\right)\right|-\mathrm{E}_{i-1}\left[\ln \left|f\left(s_{(i-1) H, i H}\right)\right|\right],
$$

and their conditional variances:

$$
\eta_{i}=\operatorname{Var}_{i-1}\left[e_{i}\right],
$$

are readily evaluated using the Kalman filter. ${ }^{4}$ When the measurement equation errors are not Gaussian, maximum likelihood estimation is more involved because a tidy closed-form expression for the likelihood, such as equation (9), does not exist in general. Therefore, the evaluation and maximization of the likelihood is much more challenging. Related, in the nonGaussian case the prediction errors $e_{i}$ produced by the Kalman filter are merely linear projection errors, not conditional expectation errors, because in non-Gaussian settings the linear projections produced by the Kalman filter do not in general coincide with the conditional expectations.

Nevertheless, maximizing the Gaussian likelihood function (9) can yield consistent parameter estimates even when the projection errors are not Gaussian. This approach is called

\footnotetext{
${ }^{4}$ For a good overview of the Kalman filter, see Hamilton (1994).
} 
Gaussian quasi-maximum likelihood estimation (QMLE). The benefits of Gaussian quasimaximum likelihood estimation are its simplicity and consistency. Its drawbacks are that the estimates are inefficient, even asymptotically, and more importantly that its small-sample properties are suspect. ${ }^{5}$ Intuitively, the further the distribution of the projection errors $\varepsilon$ is from normality, the more severe are the problems with Gaussian quasi-maximum likelihood estimation. Of course, the distribution of the projection errors is application-specific, which means that the quality of the Gaussian quasi-maximum likelihood approach can ultimately only be assessed through Monte Carlo experiments.

\section{Properties of Log Absolute or Squared Returns as Volatility Proxies}

The stochastic volatility literature primarily uses absolute or squared returns as volatility proxies. ${ }^{6}$ The continuously compounded return over the $i$ th interval is just the difference between the log asset prices at times $(i+1) H$ and $i H$. Thus, the traditional log volatility proxy is:

$$
\ln \left|f\left(s_{i H,(i+1) H}\right)\right|=\gamma \ln \left|s_{(i+1) H}-s_{i H}\right|=\gamma \ln \sigma_{i H}+\gamma \ln \left|s_{(i+1) H}^{*}-s_{i H}^{*}\right|,
$$

where $\gamma=1$ or $\gamma=2$, depending on whether we consider absolute or squared returns. Because $\gamma$ only scales the volatility proxy, and hence does not affect the distribution of the measurement equation errors, we focus exclusively, but without loss of generality, on absolute returns. That is, throughout the remainder of the paper we set $\gamma=1$.

\footnotetext{
${ }^{5}$ Note also that, quite apart from whether the model parameters are efficiently estimated, in non-Gaussian state-space models the Kalman filter generally produces inefficient filtered and smoothed extractions of the latent state vector. In particular, in non-Gaussian stochastic volatility applications the Kalman filter delivers volatility inferences that are merely best linear unbiased, not minimum variance unbiased. The two sets of inferred volatilities can diverge greatly even when the true parameters of the model are known.

${ }^{6}$ For a good survey, see Ghysels, Harvey, and Renault (1996).
} 
The second equality of equation (12) formally requires that the log security price is a martingale, so that it is homogeneous in volatility. However, this assumption is not too troubling because over sufficiently small sampling intervals $H$, such as a day or even a week, the price drift of most securities is negligible. In fact, from a statistical perspective, the assumption is likely to be helpful. By using a drift estimator that always takes the value zero we inject only a small bias, to the extent that the true drift differs slightly from zero, but we greatly reduce the variance relative to other estimators.

It is by now well known that the conditional distribution of log absolute or squared returns is far from Gaussian. Jacquier, Polson, and Rossi (1994), Andersen and Sorensen (1997), and Kim, Shephard, and Chib (1998) argue that, as a result, Gaussian quasi-maximum likelihood estimation with these traditional volatility proxies is highly inefficient and often severely biased in finite samples. Indeed, the relevant parts of our own Monte Carlo results, which we present in the next section, confirm their conclusions.

To deepen our theoretical understanding of why the conditional normality assumption for $\log$ absolute or squared returns fails, we examine the distribution of the log absolute value of a driftless Brownian motion $x$, with origin $x_{0}=0$ and constant diffusion coefficient $\sigma$, over an interval of finite length $\tau .^{7}$ Karatzas and Shreve (1991) characterize the distribution of the absolute value of a Brownian motion. A simple transformation of their result reveals that the distribution of the $\log$ absolute value is:

$$
\operatorname{Prob}\left[\ln \left|x_{\tau}\right| \in d y\right]=\frac{2 \mathrm{e}^{y}}{\sigma \sqrt{\tau}} \varphi\left(\frac{\mathrm{e}^{y}}{\sigma \sqrt{\tau}}\right) d y,
$$

\footnotetext{
${ }^{7}$ The assumption $x_{0}=0$ allows us to interpret $x_{\tau}$ directly as a continuously compounded return. $-10-$
} 
where $\varphi$ denotes a standard normal density.

From this distribution, we can compute the mean, standard deviation, skewness, and kurtosis of $\ln \left|x_{\tau}\right|$, which we present in the first row of Table I. Notice that different values of $\sigma$ and $\tau$ affect only the mean, not the variance, skewness, or kurtosis of log absolute returns. In other words, those parameters determine the location, but not the shape, of the distribution. Without loss of generality then, we graph in Figure 1a the distribution of $\ln \left|x_{\tau}\right|$ with both $\sigma$ and $\tau$ set to one. For comparison, we also plot a Gaussian density with matching mean and variance.

Table I and Figure 1a clearly demonstrate that the distribution of log absolute returns is far from Gaussian. The skewness and kurtosis of $\ln \left|x_{\tau}\right|$ are -1.5 and 6.9 , in sharp contrast to the values of 0.0 and 3.0 corresponding to normality. The intuition of this result is that both positive and negative returns close to zero, observations that are "inliers" of the return distribution, become large negative outliers of the distribution of log absolute returns. ${ }^{8}$

\section{E. Properties of the Log Range as a Volatility Proxy}

Now consider using the range as volatility proxy, where the range over the $i$ th interval is defined as the difference between the security's highest and lowest log price between times $i H$ and $(i+1) H$. Formally, consider use of the following log volatility proxy:

\footnotetext{
${ }^{8}$ The use of log absolute returns is even more problematic in empirical work on high-frequency data, because returns can be exactly zero with positive probability, due to the discreteness in prices. In that case, which arises not infrequently in practice, the logarithm of absolute returns is undefined and the quasi-maximum likelihood approach fails. Various ad hoc procedures, such as adding a small constant to the absolute returns, have been devised to skirt this problem (e.g., Breidt and Carriquiry, 1996).
} 


$$
\begin{aligned}
\ln \left|f\left(s_{i H,(i+1) H}\right)\right| & =\ln \left(\sup _{i H<t \leq(i+1) H^{-}} s_{t} \inf _{i H<t \leq(i+1) H^{\prime}} s_{t}\right) \\
& =\ln \sigma_{i H}+\ln \left(\sup _{i H<t \leq(i+1) H^{-}} s_{t}^{*} \inf _{i H<t \leq(i+1) H^{*}} s_{t}^{*}\right) .
\end{aligned}
$$

For the second equality we require again that the log price is homogeneous in volatility (i.e., that it is a martingale). ${ }^{9}$ We drop the absolute value signs because the range cannot be negative.

The log range is superior as a volatility proxy to log absolute or squared returns for two reasons. First, it is more efficient, in the sense that the variance of the measurement errors associated with the log range is far less than the variance of the measurement errors associated with $\log$ absolute or squared returns. Second - and this is a central insight of this paper - the log range is very well approximated as Gaussian. On both counts, the log range is an attractive volatility proxy for Gaussian quasi-maximum likelihood estimation of stochastic volatility models.

Let us first discuss in more detail the superior efficiency of the log range. The intuition is simple: on days when the security price fluctuates substantially throughout the day but, by chance, the closing price is close to the opening price, the absolute or squared return indicates low volatility despite the large intraday price fluctuations. The range, in contrast, reflects the intraday price fluctuations and therefore indicates correctly that the volatility is high.

\footnotetext{
${ }^{9}$ Instead of assuming a zero drift, we can perform a change of variable from the Brownian motion to a Brownian bridge (e.g., Doob, 1949; Feller, 1951). The distribution of the log range of the Brownian bridge is nearly identical to that of the log range of the corresponding Brownian motion. However, the Brownian bridge is by construction independent of the drift. See Alizadeh (1998) for details.
} 
The mathematics underlying the superior efficiency of the log range is less simple, but nevertheless standard. Specifically, consider again a driftless Brownian motion $x$, with origin $x_{0}=0$ and constant diffusion coefficient $\sigma$, over an interval of finite length $\tau$. Feller (1951) derives the distribution of the range, and a simple transformation of his result reveals that the distribution of the log range is:

$$
\operatorname{Prob}\left[\ln \left(\sup _{0<t \leq \tau} x_{t}-\inf _{0<t \leq \tau} x_{t}\right) \in d y\right]=8 \sum_{k=1}^{\infty}(-1)^{k-1} \frac{k^{2} \mathrm{e}^{y}}{\sigma \sqrt{\tau}} \varphi\left(\frac{k \mathrm{e}^{y}}{\sigma \sqrt{\tau}}\right) d y \text {. }
$$

Although this distribution is expressed as an infinite series, it is straightforward to compute its moments after suitably truncating the infinite sum. In the second row of Table I we report the mean and standard deviation. The superior efficiency of the log range, relative to the $\log$ absolute return, emerges clearly. Both proxies move one-for-one with log volatility on average, but the standard deviation of the log range is approximately one fourth the standard deviation of the $\log$ absolute return.

The efficiency of the range as a volatility measure has been appreciated implicitly for decades in the business press, which routinely reports high and low prices and sometimes displays high-low-close or candlestick plots. Range-based volatility estimation has also featured in the academic literature at least since Parkinson (1980), who proposes and rigorously analyzes the use of the range for estimating volatility in a constant volatility setting. Since then, Parkinson's estimator has been improved in several ways, including combining the range with 
opening and closing prices (e.g., Garman and Klass, 1980; Beckers, 1983; Ball and Torous, 1984; Rogers and Satchell, 1991; Yang and Zhang, 2000). ${ }^{10}$

Let us now discuss in more detail the approximate normality of the log range, or equivalently, the approximate log-normality of the range. This aspect of the range is not particularly intuitive, and it is certainly not widely appreciated. Nevertheless, it is a fact. The second row of Table I shows that the skewness and kurtosis of the log range are 0.17 and 2.80, respectively. These values are very close to the corresponding values of zero and three for a normal random variable, and they represent a sharp contrast to the earlier-presented skewness and kurtosis of the log absolute return. In Figure $1 \mathrm{~b}$ we plot the density of the log range (15), with $\sigma$ and $\tau$ set to one, together with a Gaussian density with matching mean and variance, which makes visually clear the remarkable near-normality of the distribution of the log range.

\section{F. Robustness of the Range to Market Microstructure Noise}

Thus far we have emphasized the desirable efficiency and normality properties of the range.

Here we investigate a third and intriguing property of the range, which is of independent interest and which links nicely to a central literature in high-frequency finance: robustness to certain types of market microstructure effects. ${ }^{16}$ To illustrate the robustness of the range to market microstructure effects, we compare the properties of the range to those of realized volatility,

\footnotetext{
${ }^{10}$ Although including the opening and closing prices can improve the estimation of volatility in principle, the gains are not necessarily realized in practice. In particular, Brown (1990) argues against the inclusion of the opening and closing prices on the grounds that they are highly influenced by microstructure effects, such as the lack of trading at the close or "market on the close" orders that have a disproportionate effect on the closing price. Furthermore, experimentation by Alizadeh (1998) reveals little theoretical efficiency gain from combining the range with the opening and closing prices. Thus, we do not pursue the idea in this paper.

${ }^{16}$ For a good empirically-oriented overview of market microstructure effects in security prices and returns, see Hasbrouck (1996).
} 
another highly efficient volatility proxy, in the presence of bid-ask bounce, a well-known and important source of market microstructure noise. Both the daily range and daily realized volatility use intraday data, but they process this information in very different ways and ultimately exhibit different degrees of robustness to market microstructure noise.

The concept of realized volatility has been used productively by French, Schwert, and Stambaugh (1987), Schwert (1989), and Andersen, Bollerslev, Diebold, and Ebens (2001), and is formally justified by Andersen, Bollerslev, Diebold, and Labys (2001). Realized volatility is nothing more than the sum of squared high-frequency returns over a given sampling period. For example, we calculate a daily realized volatility series by summing over each day a sequence of squared intraday returns (e.g., five-minute returns). If log security prices evolve as a diffusion and if returns are sampled sufficiently frequently, then the realized volatility is a more efficient volatility proxy than the range, because it becomes arbitrarily close to the true volatility as the sampling frequency increases. In particular, Andersen and Bollerslev (1998) show that, under such ideal conditions, the daily range is about as efficient a volatility proxy as the realized volatility based on returns sampled every four hours.

However, market microstructure can a have large impact on observed high-frequency prices and returns. For example, in the presence of a bid-ask spread, the observed price is a noisy version of the true price because it effectively equals the true price plus or minus half the spread, depending on whether a trade is buyer- or seller-initiated. Because transactions tend to bounce between buys and sells, the induced bid-ask bounce in observed prices increases the measured volatility of high-frequency returns. 
In particular, bid-ask bounce increases the volatility of high-frequency returns and hence the average size of squared high-frequency returns. By summing the squared high-frequency returns, each of which is biased upward, the realized volatility contains a cumulated and therefore potentially large bias, which becomes more severe as returns are sampled more frequently. The range, in contrast, is less likely to be seriously contaminated by bid-ask bounce. The observed daily maximum is likely to be at the ask and hence "too high" by half the spread, whereas the observed minimum is likely to be at the bid and hence "too low" by half the spread. On average, then, the range is inflated only by the average spread, which is small in liquid markets. ${ }^{17}$ The upshot is obvious: despite the fact that the range is a less efficient volatility proxy than realized volatility under ideal conditions, it may nevertheless prove superior in real-world situations in which market microstructure biases contaminate high-frequency prices and returns.

Let us illustrate matters with a simple example in the spirit of Hasbrouck (1999).

Suppose that the true log price $s_{t}$ evolves as a random walk, $s_{t}=s_{t-1}+u_{t}$, with $u_{t} \sim \operatorname{NID}\left[0, \sigma_{u}^{2}\right]$. Let the bid price be $B_{t}=$ floor $\left[S_{t}-\right.$ ticksize $]$, and let the ask price be $A_{t}=$ ceiling $\left[S_{t}+\right.$ ticksize $]$, where $S_{t}=\exp \left(s_{t}\right)$ is the true price. We then take the observed price as $S_{t}^{o b s}=B_{t} q_{t}+A_{t}\left(1-q_{t}\right)$, where $q_{t}=$ Bernoulli[1/2]. Hence the observed price fluctuates randomly between the bid and the ask. $^{18}$

In Figure 2, we show a typical one-day sample path of 289 simulated five-minute true and observed prices. Following Hasbrouck (1999), we use $S_{0}=\$ 25$, ticksize $=\$ 1 / 16$, and $\sigma_{u}=0.0011$,

\footnotetext{
${ }^{17}$ Moreover, one could readily perform a bias correction by subtracting the average spread from the range. We thank Joel Hasbrouck for this observation.

${ }^{18}$ We could go even further and induce negative autocorrelation in $q_{t}$ by taking $q_{t}=$ Bernoulli $\left[1 / 2+\theta_{0}\right]$ if $q_{t-1}=0$ and $q_{t}=$ Bernoulli $\left[1 / 2-\theta_{1}\right]$ if $q_{t-1}=1$, for $\theta_{0}, \theta_{1}>0$. Doing so, however, would only strengthen the results.
} 
which implies an annualized thirty percent return volatility (standard deviation), assuming 250 trading days per year. The population daily return volatility is 1.87 percent (i.e., $100 * \sqrt{288 \sigma_{u}^{2}}$ ), and the realized volatility calculated using the true returns is a close 1.81 percent. In contrast, the realized volatility based on the much noisier observed returns is an inflated 6.70 percent! The market microstructure noise in the observed returns also affects the range-based volatility estimator insofar as the observed daily maximum and minimum differ from their true counterparts, resulting in an observed range that is greater than the true range, but the effect is comparatively minor relative to the overall daily movement of the true and observed prices. For the true and observed price paths on this "day," the range-based volatility estimates are 1.54 percent and 1.79 percent, respectively. ${ }^{19}$

\section{Monte Carlo Analysis}

The diffusion theory sketched above shows that the log range is a less noisy volatility proxy than $\log$ absolute or squared returns and that the distribution of the log range is approximately Gaussian, in stark contrast to the skewed and leptokurtic distribution of the traditional returnbased volatility proxies. Both of these findings suggest that Gaussian quasi-maximum likelihood estimation with the log range as volatility proxy is highly efficient, not only relative to quasimaximum likelihood estimation with the traditional return based volatility proxies, but also relative to exact maximum likelihood estimation.

We now use a Monte Carlo experiment to compare quasi-maximum likelihood estimation with the log range as volatility proxy to both quasi- and exact maximum likelihood estimation with the log absolute return as volatility proxy. In particular, we generate 5000 samples of

\footnotetext{
${ }^{19}$ We use Parkinson's (1980) volatility estimator of 0.361 times the squared range.
} 
$T=1000$ or 500 daily observations of the two volatility proxies, where each daily price path is generated by $N=1000,100$, or 50 intraday price moves. For every sample, we then perform quasi-maximum likelihood estimation of the stochastic volatility model (2) with either the log range or the log absolute return as volatility proxy. For comparison, we also perform exact maximum likelihood estimation with the log absolute return, where we evaluate the likelihood function using the simulation approach of Sandmann and Koopman (1998).

We simulate the daily price paths from the following Euler approximation of the discretized stochastic volatility model (4)-(5):

$$
\begin{gathered}
s_{t}=s_{t-\Delta t}+\sigma_{i H} \varepsilon_{s t} \sqrt{\Delta t} \\
\ln \sigma_{(i+1) H}=\ln \bar{\sigma}+\rho_{H}\left(\ln \sigma_{i H}-\ln \bar{\sigma}\right)+\beta \varepsilon_{v i} \sqrt{H},
\end{gathered}
$$

for $i H<t \leq(i+1) H$, where $\varepsilon_{s t}$ and $\varepsilon_{v i}$ are independent $\mathrm{N}[0,1]$ innovations. The discrete time increment $\Delta t$, a small fraction of the discrete sampling interval $H$, approximates the continuous time $d t$. We set $H=1 / 257$ and $\Delta t=H / N$, which corresponds to daily data generated by $N$ trades per day, and we set $\alpha=3.855, \ln \bar{\sigma}=-2.5$, and $\beta=0.75$, which implies a volatility process with a daily autocorrelation of $\rho_{H}=0.985$, an annualized average volatility of 8.51 percent, and a coefficient of variation of $0.28 .{ }^{20}$ These volatility dynamics are broadly consistent with our subsequent empirical results for five major currencies as well as with the literature on stochastic volatility.

\footnotetext{
${ }^{20}$ Following Jacquier, Polson, and Rossi (1994) we interpret the volatility of $\log$ volatility parameter $\beta$ through the coefficient of variation, $\left(\operatorname{Var}\left[\sigma_{t}\right] / \mathrm{E}^{2}\left[\sigma_{t}\right]\right)^{1 / 2}$.
} 


\section{A. Parameter Estimates}

Tables II and III summarize the sampling distributions of the three estimators of $\rho_{H}$, $\beta$, and $\ln \bar{\sigma}$ for $T=1000$ and $T=500$ daily observations of the volatility proxies, respectively. Each table is made up of three parts corresponding to $N=1000, N=100$, and $N=50$ trades per day.

Consider first the case $T=1000$ and $N=1000$ in Table IIA. Using the absolute return as volatility proxy, the average quasi-maximum likelihood estimate of $\rho_{H}$ is 0.95 , compared to an average estimate of 0.98 using the range as volatility proxy and the true value of 0.985 . Even more strikingly, the root mean squared errors (RMSE) of the estimates are 0.14 and 0.01 , respectively. Clearly, using the range instead of the absolute return as volatility proxy produces quasi-maximum likelihood estimates that are both less biased and less variable.

The performance difference between the two quasi-maximum likelihood estimators is even more impressive for the volatility of $\log$ volatility parameter, $\beta$. The average estimate using the $\log$ absolute return is 1.08 with an RMSE of 1.18 . In contrast, the average estimate using the log range is 0.8 , close to the true value of 0.75 , with an RMSE of only 0.12 .

In contrast, the results for the mean $\log$ volatility $\ln \bar{\sigma}$ are basically identical. Intuitively, this is because the average level of volatility is directly identified by the unconditional mean of the volatility proxies. The estimates of the average level of volatility are thus relatively insensitive to the statistical properties of the measurement equation errors.

In Figure 3, we illustrate graphically the very different finite-sample properties of the two quasi-maximum likelihood estimators. The first three plots of the first two rows show the sampling distributions of the parameter estimates using the log absolute return and the log range as volatility proxy, respectively. The drastic efficiency gains from using the range are 
immediately apparent. ${ }^{21}$ Furthermore, the sampling distributions of the estimates of $\rho$ and $\beta$ for the log absolute return are severely skewed, which implies that the usual Gaussian inferences based on asymptotic standard errors are not trustworthy. In contrast, the distributions of the corresponding estimates using the log range are very close to Gaussian.

The results thus far indicate that quasi-maximum likelihood estimation with the log range as volatility proxy is far more efficient than with the log absolute return as volatility proxy. This efficiency gain stems from the range being a much less noisy volatility measure as well as from the log range being approximately Gaussian. To separate these two effects, we now compare the range-based quasi-maximum likelihood estimator to the exact maximum likelihood estimator for absolute returns. If the only benefit from using the range is its approximate normality, the results for the range-based quasi-maximum likelihood estimator should be very similar to the results for the exact maximum likelihood estimator for absolute returns. If, however, the information about intraday volatility revealed by the range but not by absolute or squared returns is useful in the estimation of the model, the sampling properties of the range-based quasi-maximum likelihood estimator could well dominate the sampling properties of the exact maximum likelihood estimator for absolute returns. ${ }^{22}$

Comparing the second and third panel of Table IIA reveals that much but not all of the efficiency gain from using the log range as volatility proxy is attributed to the approximate normality of the log range (see also the second and third rows of Figure 3 for a graphical

\footnotetext{
${ }^{21}$ Notice the different scales of the second plot in each row. The horizontal axes of the second plot in the second and third rows correspond to the region between the two vertical lines in the second plot of the first row.

${ }^{22}$ Alternatively, we could compare the properties of the range-based quasi-maximum likelihood estimator to those of the exact maximum likelihood estimator for the range. However, given the near-normality of the $\log$ range, the difference in performance between these two estimators would be minimal.
} 
representation of the results). In terms of bias, the range-based quasi-maximum likelihood estimator and the exact maximum likelihood estimator for absolute returns perform equally well. However, the RMSEs of the range-based estimates of $\rho$ and $\beta$ are significantly smaller than for the corresponding exact maximum likelihood estimates ( 0.012 versus 0.016 for $\rho$ and 0.122 versus 0.207 for $\beta$ ). This demonstrates that the information about intraday volatility contained in the range is a crucial ingredient to the success of the range-based estimator.

Because the return-based estimators do not utilize intraday data, their sampling distributions are independent of the number of trades per day $N$. The properties of the rangebased estimator, in contrast, depend on the level of trading activity. In particular, when there are only a few trades per day, the observed range can be far from the true range of the underlying price process and, as a result, range-based volatility estimates can substantially deviate from the true volatility. To examine the robustness of the range-based estimator to less frequent trading, we show in parts B and $\mathrm{C}$ of Table II results for $N=100$ and $N=50$ trades per day, respectively.

The general pattern is that as $N$ decreases the range-based estimators of both $\rho$ and $\beta$ become more biased ( $\rho$ is downward biased while $\beta$ is upward biased) and less precise. More specifically, with 100 trades per day the performance of the range-based quasi-maximum likelihood estimator is comparable to that of the exact maximum likelihood estimator with log absolute returns. Even with only 50 trades per day, it still dominates the quasi-maximum likelihood estimator with log absolute returns, in terms of both bias and RMSE. Further experimentation with the trading frequency reveals that range-based estimation is inferior to returns-based estimation only when there are less than 10 trades per day. (Detailed results are available upon request). 
Table III presents the Monte Carlo results for $T=500$ daily observations of the volatility proxy. It appears from the table that the range-based estimator is less sensitive to the reduction in the sample size than the return-based estimators. The RMSEs of the range-based estimators of $\rho$ and $\beta$ increase by 97 percent (from 0.012 to 0.023 ) and 48 percent (from 0.122 to 0.180 ), respectively. The corresponding percentage increases in the RMSEs for the quasi- and exact maximum likelihood estimators using absolute returns are significantly larger, with 102 and 225 percent for $\rho$ and 83 and 96 percent for $\beta$.

Interestingly, increasing the sample size to $T=5000$ or even 10000 does not dramatically improve the performance of the quasi-maximum likelihood estimator with log absolute returns. (Detailed results are available upon request). In particular, the estimator of $\beta$ remains severely biased and extremely imprecise.

Finally, we repeat the Monte Carlo analysis allowing the volatility to vary throughout the day to verify that the discretization (4)-(5) of the continuous time model (2) does not substantially affect the estimator. The results (available upon request) are virtually identical to those in tables II and III, which confirms that, at least for the parameterization of the model we consider, the effect of the discretization is negligible.

\section{B. Volatility Extraction}

Once the model has been estimated, the Kalman filter can be used to extract the latent stochastic volatility series. $^{23}$ The Kalman filter produces linear projections, which coincide with conditional expectations only under the assumption of joint normality. Therefore, the extraction

\footnotetext{
23 The Kalman filter produces one-step ahead forecasts $\mathrm{E}\left[\ln \sigma_{t} \mid I_{t-1}\right]$, concurrent estimates $\mathrm{E}\left[\ln \sigma_{t} \mid I_{t}\right]$, and smoothed extractions $\mathrm{E}\left[\ln \sigma_{t} \mid I_{T}\right]$. Throughout this section, we use the smoothed extractions, but we checked that our results are not changed significantly if instead we use one-step ahead forecasts.
} 
of the latent volatilities is best unbiased when using a Gaussian volatility proxy, whereas the extraction is merely best linear unbiased when using a non-Gaussian volatility proxy. This implies that there are two reasons to expect the volatility extraction with the log range to dominate the extraction with the log absolute return. First, the range-based parameter estimates are more accurate. Second, even for the same parameter values, the efficiency of the range as a volatility proxy and the approximate normality of the log range yield more accurate volatility extractions.

With this in mind, we summarize in the middle and right sections of tables II and III the sampling distributions of the mean extraction error $\mathrm{E}\left[\hat{\sigma}_{t}-\sigma_{t}\right]$ and the root mean squared (RMS) extraction error $\mathrm{E}\left[\left(\hat{\sigma}_{t}-\sigma_{t}\right)^{2}\right]^{1 / 2}$. Because the volatility can take on quite different values in a given sample as well as across samples, we also report the distributions of the mean percent extraction error $\mathrm{E}\left[\left(\hat{\sigma}_{t}-\sigma_{t}\right) / \sigma_{t}\right]$ and the RMS percent extraction error $\mathrm{E}\left[\left(\left(\hat{\sigma}_{t}-\sigma_{t}\right) / \sigma_{t}\right)^{2}\right]^{1 / 2}$. In the middle section of each table, we compute the volatility extractions using the estimated parameters. In the right sections we instead feed the Kalman filter the true parameters.

Not surprisingly, among the quasi-maximum likelihood estimators the range-based estimator is superior. Consider, for example, the results for $T=1000$ and $N=1000$ in Table IIA. Both volatility extractions appear unbiased, but the range-based extraction is much more efficient. With estimated parameters, the log absolute return produces a RMS extraction error of 1.8 percent or 22 percent relative to the level of volatility (the average level of volatility is 8.2 percent). Using the log range as volatility proxy, the RMS extraction error is only 1.2 percent or 14 percent in relative terms. With the true parameters, both estimators become more accurate, but their relative performance remains the same. 
Comparing the range-based quasi-maximum likelihood extractions to the results for the exact maximum likelihood estimator for absolute returns, we again notice that the information about intraday volatility contained in the range is important. The exact maximum likelihood estimates are based on a non-Gaussian filter, meaning that the extractions are actually conditional expectations and not just linear projections produced by the Kalman filter. Therefore, the differences between the range-based quasi-maximum likelihood extractions with known parameters and the corresponding exact maximum likelihood results for absolute returns are not induced by problems with the estimator (such as sub-optimal filtering) but are simply due to the superior informational efficiency of the range.

Comparing the range-based extraction errors across the three parts of Table II reveals an interesting pattern. With estimated parameters, the distribution of the RMS extraction error is relatively unaffected by the number of trades per day. With the true parameters, in contrast, the average RMS extraction error increases from one percent with $N=1000$ trades per day to 1.25 percent with $N=50$ trades per day.

Finally, putting Tables II and III side-by-side shows that as the number of observations decreases and, as a result, the small sample biases of the parameter estimates become more severe, the extraction errors with estimated parameters obviously increase. ${ }^{24}$ Because, as we discovered above, the return-based parameters estimates are more sensitive to the smaller sample size, the return-based volatility extractions also become relatively more noisy.

C. Robustness of the Range to Market Microstructure Noise

\footnotetext{
${ }^{24}$ Notice that the volatility extractions with the true parameters do not depend on the sample size $T$. Therefore, the extraction errors with known parameters are omitted from Table III.
} 
In Section II.F above, we conjectured that the range-based volatility estimator is robust to microstructure noise, in contrast to other popular volatility estimators such as realized volatility, and we substantiated this conjecture with an example based on a single day of simulated prices. We now perform a more systematic analysis based on repeated samples. Using the same parameter values as before, we simulate one day of five-minute true and observed prices (289 observations), and we calculate both realized and range-based daily volatility estimates, based upon both true and observed prices, using a variety of underlying sampling frequencies (5minute, 10-minute, 20-minute, 40-minute, 1 hour and 20 minutes, 3 hour, 6 hour, and 12 hour). We repeat this 100,000 times, and we report means, standard deviations and root mean squared errors of the corresponding distributions in Table IV. For subsequent reference, recall that our design implies that the population volatility (standard deviation) of true daily returns is in fact fixed at 1.87 percent, which implies an annualized volatility of thirty percent.

First consider estimating volatility using the true underlying price series. In this case, realized volatility is unbiased regardless of the return interval, and its standard deviation decreases monotonically toward zero as the return interval shrinks. In contrast, range-based volatility is biased downward, regardless of the return interval, because the range on a discrete grid can only be less than the range of the true continuous sample path. As the sampling interval shrinks, this bias decreases monotonically. Interestingly, however, the standard deviation of the range-based estimator increases monotonically as the sampling interval shrinks. By the time we arrive at 5-minute sampling, the efficiency (RMSE) of range-based volatility is between that of realized volatility computed using 3-hour and 6-hour returns, which accords with the results of Andersen and Bollerslev (1998). 
All told, realized volatility clearly dominates range-based volatility when based on the true underlying price. The efficiency of realized volatility is superior regardless of the sampling interval, and the efficiency of realized volatility relative to that of range-based volatility increases without bound as the return interval shrinks.

Now we consider the effects of the market microstructure noise. The bid-ask bounce biases realized volatility upward, and the bias increases monotonically as the underlying return interval shrinks. To make matters worse, the variability of realized volatility stays high as the return interval shrinks, because the benefits of using of high-frequency data are eventually overpowered by the harmful effects of market microstructure noise. All of these effects are distilled in the RMSE of realized volatility, which spikes sharply upward as the return interval shrinks.

Bid-ask bounce affects range-based volatility differently. In particular, the discreteness associated with long return intervals biases range-based volatility downward slightly, but the bidask bounce tends to bias it upward slightly. The two biases trade off against each other, often partly canceling, typically producing very good performance of range-based volatility in the presence of microstructure noise.

In summary, the tables are clearly turned when calculations are based on observed rather than true underlying returns: range-based volatility performs admirably relative to realized volatility, and the efficiency of range-based volatility relative to that of realized volatility increases as the return interval shrinks. We highlight certain aspects of the results in Figures 4 and 5, which show the distributions of realized volatility and range-based volatility for the true and observed price paths, for sampling intervals of 5, 20, and 80 minutes. In the case of true 
prices, the performance of range-based volatility is approximately unchanged as we move from 80-minute to 5-minute sampling, whereas the performance of realized volatility improves sharply. In the case of observed prices, the performance of range-based volatility deteriorates moderately as we move from 80-minute to 5-minute sampling, whereas the performance of realized volatility deteriorates sharply.

\section{Exchange Rate Volatility Dynamics}

The nature of exchange rate volatility dynamics has important implications for currency derivative pricing, portfolio allocation, and risk management. Here we use our simple rangebased maximum likelihood approach to shed light on the nature of those volatility dynamics, with an eye toward the number and interpretation of the latent factors that drive volatility. We estimate stochastic volatility models for the U.S. dollar price of five actively-traded currencies: the British pound, Canadian dollar, Deutsche Mark, Japanese yen, and Swiss franc. We construct the volatility proxies from daily high, low, and closing futures prices. Before we turn to the estimates of the model, we first tabulate some statistics describing the salient aspects of the volatility proxies.

\section{A. Data}

We use daily high, low, and closing (3pm EST) prices of currency futures contracts traded on the International Monetary Market, a subsidiary of the Chicago Mercantile Exchange, from January 1978 through December 1998 (5284 observations). ${ }^{25}$ A currency futures contract represents delivery of the currency on the second Wednesday of the following March, June, September, or December. Each day there are at least three futures contracts with different quarterly delivery

\footnotetext{
${ }^{25}$ The data source is FAME Information Services.
} 
dates traded on each currency. We use futures prices from the front-month contract, which is the contract closest to delivery and with at least ten days to delivery. This front-month contract is typically the most actively traded futures contract.

There are several advantages to using futures, as opposed to spot, exchange rate data. First, all futures prices (including the daily high and low) result from open outcry, so that all transactions are open to the market and orders are filled at the best price. Currency spot market trading, in contrast, is based on bilateral negotiation between banks, and any one particular executed price is not necessarily representative of the overall market conditions. Second, the closing, or "settlement," futures price is based on the best sentiment of the market at the time of close (3pm EST, after which spot market trading declines) and is widely scrutinized, because it is used for marking to market all account balances. Therefore, the futures closing price is likely to be a very accurate measure of the "true" market price at that time. Finally, futures returns are the actual returns from investing in a foreign currency, whereas spot "returns" are less meaningful, unless one accounts for the interest rate differential between the two countries.

A potential disadvantage of using futures prices is that the futures volatility may differ from the spot volatility and, furthermore, that the difference between the two volatilities may depend on the time-to-maturity of the contract. For exchange rates, the cash-and-carry relationship $F_{t}^{\tau}=S_{t} \exp \left(\Delta r_{t}^{\tau} \tau\right)$, where $F_{t}^{\tau}$ denotes the $\tau$-period futures price and $\Delta r_{t}^{\tau}$ is the $\tau$ period interest rate differential between the two countries, implies the approximate daily variance decomposition $\operatorname{Var}\left[f_{(i+1) H}^{\tau-H}-f_{i H}^{\tau}\right] \approx \operatorname{Var}\left[s_{(i+1) H}-s_{i H}\right]+\tau^{2} \operatorname{Var}\left[\Delta r_{(i+1) H}^{\tau-H}-\Delta r_{i H}^{\tau}\right]$, where we ignore the covariance between the daily spot returns and the daily changes in the interest rate differential. Suppose the annualized spot volatility is ten percent and the annualized volatility of changes in 
the interest rate differential is four percent, which are both realistic numbers. Then for a 45 day contract (the average maturity in our sample), the difference between the annualized futures and spot volatility is less than one basis point. We conclude therefore that at least for relatively short-dated contracts the difference between the futures and spot volatility is in theory negligible.

To verify this conclusion empirically, we perform two robustness checks. First, we use five-minute samples of the spot rate for the British pound, Deutsche Mark, Japanese yen, and Swiss franc from December 3, 1986 to December 1, 1998 to construct a series of spot ranges, and we regress the futures ranges on the corresponding spot ranges and a constant. All of the resulting regression slope estimates are very close to one (within 0.05 ), all intercept estimates are very close to zero (within 0.05 ), and all $R^{2}$ s are high (above 0.85 ). Second, we estimate stochastic volatility models where we add polynomial terms in the time-to-maturity of the futures contract as exogenous regressors in the measurement equation to capture any biases induced by rolling the futures every three months to the current front-month contract. The coefficients on these time-to-maturity terms are both statistically and economically negligible. (Detailed results are available upon request).

In light of the above arguments in favor of using futures prices to calculate daily ranges and returns, we do so from this point onward. In Table V we present statistics summarizing the distributions of $\log$ absolute returns and the log range for each of the five currencies. The superior efficiency of the log range as a volatility proxy emerges not only in terms of its smaller standard deviation stressed thus far, but also in terms of its time-series dynamics. In particular, the large and slowly-decaying autocorrelations of the log range clearly reveal strong volatility 
persistence for each exchange rate, in sharp contrast to the spuriously small autocorrelations of $\log$ absolute returns, whose measurement error masks the persistence in volatility.

\section{B. One-Factor Stochastic Volatility Model Estimates and Residual Diagnostics}

The left panel of Table VI reports estimates of the traditional one-factor stochastic volatility model (4)-(5) for the five currencies. The absolute return-based estimates closely accord with other estimates of this model in the literature. The range-based estimates, in contrast, are at odds with both the return-based estimates and the results in the literature. In particular, the estimated volatility persistence parameter $\rho$ ranges from 0.62 to 0.85 , with four of the five estimates below 0.75 , compared to typical estimates of 0.80 to 0.99 . Equally puzzling at first sight, the rangebased estimate of the volatility of $\log$ volatility parameter $\beta$ is about three to five times larger than the corresponding absolute return-based estimates (implying coefficients of variation that range from 0.40 to $0.96 \mathrm{vs}$. from 0.20 to 0.42 ).

Because the differences between the return- and range-based estimates of $\rho$ and $\beta$ are exactly opposite in sign to the relative small-sample biases of the quasi-maximum likelihood estimators in our Monte Carlo analysis (in small samples the absolute return based estimate of $\rho$ is more downward biased and that of $\beta$ is more upward biased), we speculate that these differences are not attributed to a problem with the estimators, but are rather due to the two estimators reacting differently to model misspecification.

To assess model misspecification more carefully, in Table VII we present diagnostics for the measurement equation residuals, $\varepsilon$, for the one-factor model. ${ }^{26}$ Indeed, the residual

\footnotetext{
${ }^{26}$ The measurement equation residuals diagnostics are the same statistics computed earlier for the observed $\log$ absolute returns and log ranges.
} 
diagnostics for the range-based estimator indicate serious problems with the one-factor model specification. While the residuals are clearly less persistent than the log range itself (compare the autocorrelations in Tables VII and V), substantial residual serial correlation remains. Effectively, the one-factor stochastic volatility model adequately accounts for the volatility correlation at lag one, but not at longer lags, which results in a humped-shaped residual autocorrelation function.

The misspecification of the one-factor model can be seen in another way. To obtain the estimates in Table VI, we set the standard deviation of the measurement equation disturbances to 0.29 , following the results in Table I. Alternatively, however, we can estimate the standard deviation of the measurement errors along with the other parameters, and when we do so, we typically obtain a much larger estimate of $\rho$. Consider, for example, the British pound. When we set the standard deviation of the measurement errors to 0.29 , we obtain $\hat{\rho}=0.66$, as recorded in Table VI, but when we estimate the standard deviation of the measurement errors along with the other parameters, we obtain $\hat{\rho}=0.97$ and an estimate of the standard deviation of 0.42 . The difference in maximized log likelihoods, moreover, is greater than two hundred. Hence, the measurement errors of the one-factor model are much more variable than expected if the onefactor model were correct - a standard deviation of 0.42 vs. 0.29 - which again suggests that the one-factor model is not correct. $^{29}$

\footnotetext{
${ }^{29}$ In fact, the sum of the unconditional variance of the measurement errors and the unconditional variance of the latent $\log$ volatility process exceeds the unconditional variance of the log range (from Table V), which suggests a negative correlation between log volatility and the measurement errors. In theory, of course, the measurement errors are uncorrelated with log volatility.
} 


\section{Two-Factor Stochastic Volatility Model Estimates and Residual Diagnostics}

In light of the severe deficiencies of the one-factor stochastic volatility model revealed by our range-based estimation and analysis, we move to a two-factor model, with transition equation:

$$
\ln \sigma_{(i+1) H}=\ln \bar{\sigma}+\ln \sigma_{1,(i+1) H^{+}} \ln \sigma_{2,(i+1) H},
$$

where

$$
\begin{aligned}
& \ln \sigma_{l,(i+1) H}=\rho_{l, H} \ln \sigma_{1, i H}+\beta_{l} \sqrt{H} v_{l,(i+1) H} \\
& \ln \sigma_{2,(i+1) H}=\rho_{2, H} \ln \sigma_{2, i H}+\beta_{2} \sqrt{H} v_{2,(i+1) H},
\end{aligned}
$$

and where the volatility component innovations $v_{1}$ and $v_{2}$ are contemporaneously and serially independent $\mathrm{N}[0,1]$ variates. Notice that the means of $\ln \sigma_{1,(i+1) H}$ and $\ln \sigma_{2,(i+1) H}$ are not separately identifiable. Hence we include a mean $\ln \bar{\sigma}$ in (17), but not in the individual volatility factor equations (18).

When we estimate the two-factor stochastic volatility model, the results of which we report in the right panel of Table VI, we find that one factor has highly persistent dynamics and the other has transient dynamics. Each factor is responsible for approximately half the long-run (unconditional) variance of $\log$ volatility, but the transient factor is responsible for much more of the short-run variance..$^{30}$ This result is intuitively appealing and in line with properties of volatilities estimated using very different procedures, such as the realized volatilities of Andersen, Bollerslev, Diebold, and Labys (2001), which seem to display slow persistent movement, with high-frequency noise superimposed. The residual diagnostics for the two-factor

${ }^{30} \mathrm{By}$ independence of the volatility factors, the unconditional variance of volatility is the sum of the unconditional variances of the two volatility factors. The unconditional variance of each volatility factor, in turn, is its respective innovation variance divided by one minus its squared serial correlation coefficient. The unconditional variances of the volatility factors tend to be roughly equal, although for different reasons. The high serial correlation underlying the first volatility factor is responsible for relatively more of its unconditional variation than is its innovation variance, whereas the situation for the second volatility factor is just the opposite. 
models, reported in Table VIII, indicate that the two-factor models are adequate. In particular, the measurement equation residuals for the two-factor model are serially uncorrelated, in sharp contrast to those for the one-factor model.

An interesting feature of our results is that the estimated one-factor volatility persistence parameter is an average of the estimated persistence parameters from the two-factor model. To understand this finding, consider the two-factor stochastic volatility model (17)-(18). Suppose, however, that although the two-factor model is true, we fit a one-factor model, which captures only the sum of the components, $\ln \sigma_{(i+1) H}$, instead of the individual components, $\ln \sigma_{1,(i+1) H}$ and $\ln \sigma_{2,(i+1) H}$. Then the first autocovariance of $\ln \sigma_{(i+1) H}$ is:

$$
\begin{aligned}
\operatorname{Cov}\left[\ln \sigma_{(i+1) H}, \ln \sigma_{i H}\right] & =\operatorname{Cov}\left[\ln \sigma_{1,(i+1) H}+\ln \sigma_{2,(i+1) H}, \ln \sigma_{1, i H}+\ln \sigma_{2, i H}\right] \\
& =\operatorname{Cov}\left[\ln \sigma_{1,(i+1) H}, \ln \sigma_{1, i H}\right]+\operatorname{Cov}\left[\ln \sigma_{2,(i+1) H}, \ln \sigma_{2, i H}\right] \\
& =\rho_{1} \operatorname{Var}\left[\ln \sigma_{1,(i+1) H}\right]+\rho_{2} \operatorname{Var}\left[\ln \sigma_{2,(i+1) H}\right],
\end{aligned}
$$

where of course the variances are unconditional. Hence the first autocorrelation of $\ln \sigma_{(i+1) H}$ in the one-factor model is simply:

$$
\rho=\frac{\operatorname{Cov}\left[\ln \sigma_{(i+1) H}, \ln \sigma_{i H}\right]}{\operatorname{Var}\left[\ln \sigma_{(i+1) H}\right]}=\frac{\rho_{1} \operatorname{Var}\left[\ln \sigma_{1,(i+1) H}\right]+\rho_{2} \operatorname{Var}\left[\ln \sigma_{2,(i+1) H}\right]}{\operatorname{Var}\left[\ln \sigma_{1,(i+1) H}\right]+\operatorname{Var}\left[\ln \sigma_{2,(i+1) H}\right]}
$$

which is a relative variance weighted average of the first autocorrelations of the two factors. This is approximately true in the estimates. The fact that we can successfully predict the outcome of estimating a one-factor model on the basis of our estimates of the two-factor model is further evidence in favor of the two-factor model.

The range-based estimates of the one- and two-factor models are also consistent in terms of implied unconditional variances of $\log$ volatility. Given the independence of $\ln \sigma_{1}$ and $\ln \sigma_{2}$, 
the unconditional variance of $\ln \sigma$ in the one-factor model should be equal to the sum of the variances of the two factors in the two-factor model. This is approximately true.

\section{Empirical Normality of the Log Range}

Thus far we have used measurement equation residual autocorrelations to ascertain that onefactor volatility structure is inadequate, and that a two-factor structure appears adequate. The residual autocorrelations from range-based estimation clearly reveal the defects of the one- factor model, whereas the residual autocorrelations from estimation based on absolute returns do not. The superior ability to discriminate among models when using the range-based volatility proxy stems from its high efficiency.

We have argued throughout this paper, however, that range-based volatility estimation is powerful and convenient not only because of the efficiency of the range, but also because of the near-normality of the range. Hence it is of great interest to check whether our earlier theoretical and Monte Carlo assertions on normality of the log range are verified empirically. Importantly, they are. Both the moments reported in Tables VII and VIII and the histograms and quantilequantile (QQ) plots in Figure 6 provide striking verification of the theory: the measurement equation residuals for the two-factor models are virtually indistinguishable from Gaussian when we use the range-based volatility proxy. ${ }^{31}$ When volatility is proxied by absolute returns, in contrast, the measurement equation residuals are highly skewed and leptokurtic.

The empirical normality of the log range is important, because it means that the Gaussian quasi-likelihood that we maximize is in fact not a quasi-likelihood, but the true likelihood.

\footnotetext{
${ }^{31}$ A Gaussian QQ plot is simply a graph of the quantiles of a standardized distribution against the corresponding quantiles of a N[0,1] distribution. Hence if a variable is normally distributed, its Gaussian QQ plot is a straight line with a unit slope, which enables simple visual assessment of closeness to normality.
} 
Hence the large parameter estimation efficiency gains achievable are realized in practice.

Ultimately, both the efficiency and normality of the log range are important, and they interact in valuable ways. Operating in tandem, the two enable us to quickly detect and discard inadequate specifications, to settle upon a preferred specification, and to obtain easily-computed yet highly precise estimates maximum-likelihood estimates of its underlying parameters.

\section{E. What Do We Learn from the Range, and Why Two Factors?}

We have emphasized repeatedly that efficiency and normality of the range lead to simple yet highly efficient methods of estimating stochastic volatility models. Here we delve more deeply into the reasons for the success of range-based procedures. We address the question posed in the subsection title in two parts. First we consider what we learn from the range quite generally, regardless of the specific application. Second, we consider what we learn from the range specifically about exchange rate volatility dynamics.

The key to the general success of range-based estimation in both model specification and estimation is its superior information about the volatility of volatility, relative to traditional proxies such as absolute returns. A volatility model must explain two things: the autocorrelation of volatility, and the volatility of volatility. Because it is difficult to assess the volatility of volatility using traditional proxies, due to the overwhelming amount of measurement error, estimation using traditional proxies emphasizes explaining the autocorrelation of volatility and hence tends to produce models that appear well-specified in terms of small residual autocorrelations. Range-based volatility proxies, on the other hand, are contaminated by much less measurement error, and range-based estimation therefore appropriately attempts to explain not only the autocorrelation of volatility, but also the volatility of volatility. 
In the context of our investigation of exchange rate volatility dynamics, the range-based analysis points sharply toward a two-factor volatility specification. It is natural to ask why the range-based analysis clearly reveals misspecification of the one-factor model, as revealed by the obvious patterns in the residual autocorrelations, whereas analysis based on absolute returns seems to indicate adequacy of the one-factor model. The explanation is that the large amount of noise in the absolute returns masks the presence of the second, less persistent, factor. In fact, upon closer inspection we notice that both sets of one-factor models are equally misspecified, but that the misspecification is revealed along different dimensions. The misspecification of the range-based models is immediately apparent from the autocorrelations of the residuals. The misspecification of the models based on absolute returns, in contrast, is more subtle. It appears as a violation of the adding-up constraint, $\operatorname{Var}\left[\ln \left|f_{t}\right|\right]=\operatorname{Var}\left[\ln \sigma_{t}\right]+\operatorname{Var}\left[\varepsilon_{t}\right]$.

Both estimators choose the parameters $\ln \bar{\sigma}, \rho_{H}$, and $\beta$ to match two features of the data: the autocorrelation of the volatility proxy and the difference between the unconditional variance of the volatility proxy and the corresponding unconditional variance of the measurement errors from Table I. The relative importance of those features, however, differs across the volatility proxies. Specifically, the latent volatility dynamics explain less than ten percent of the unconditional variance of $\log$ absolute returns, but more than seventy percent of the variance of the log range, which is just another manifestation of the informational efficiency of the log range. The quasi-maximum likelihood estimator using log absolute returns therefore chooses parameters that explain entirely the autocorrelation of the volatility proxy, but leave unexplained half of the variance of $\log$ absolute returns that is attributed to the volatility dynamics (which is very little relative to the total variance of log absolute returns). In contrast, the estimator using the log 
range chooses parameters that explain all of the variance of the volatility proxy, but leave a significant amount of autocorrelation (about half) unexplained.

It is interesting to note that the misspecification of the one-factor model is also readily revealed in a different way, by simulating data from a two-factor model and then estimating a one-factor model. The estimates of the one-factor model based on absolute returns appear reasonable, whereas the range-based estimates appear bizarre. In particular, just as in the real data, the range-based estimate of $\rho$ is too low and that of $\beta$ is too high. ${ }^{32}$ Furthermore, again just as in the real data, the residuals from the one-factor model based on absolute returns appear white, whereas the residuals from range-based estimation are highly serially correlated.

The upshot: range-based exchange rate volatility analysis makes clear that two volatility factors (one with persistent dynamics and one with transient dynamics) are needed to explain exchange rate volatility, as one-factor models are incapable of simultaneously fitting the persistence of volatility and the volatility of volatility. Situations of partially persistent and partially transient dynamics arise in many areas, and perhaps it is not surprising - and even economically appealing - that our two-factor exchange rate volatility dynamics are of that form, consistent with the idea that some news is easily interpretable by the market and hence readily and unambiguously incorporated into prices, while other news is not.

\section{F. Links to Long Memory: Structural vs. Reduced-Form Approaches}

Interestingly, our two-factor volatility structure is related to the repeated findings of longmemory fractionally-integrated volatility dynamics, as reported prominently for example in Andersen and Bollerslev (1997). In particular, as emphasized by Barndorff-Nielsen and

\footnotetext{
${ }^{32}$ Detailed results are available upon request.
} 
Shephard (2000, 2001), fractionally-integrated dynamics can be built up by superimposing Ornstein- Uhlenbeck or AR(1) processes. It seems clear, however, that multi-factor volatility "structures" are more readily interpreted and learned from than their fractionaly-integrated "reduced forms." For example, in the previous section it emerged that one of the factors was highly persistent and responsible for the autocorrelation in volatility, while the other was much less persistent and responsible for the volatility of volatility. ${ }^{33}$ This interpretability stands in sharp contrast to models of long-memory fractionally-integrated volatility dynamics, which often appears mysterious and non-intuitive.

The two-factor component structure may also produce volatility forecasts superior to those from fractionally-integrated reduced-form specifications. Suppose, for example, that volatility today is very high. The forecast of tomorrow's volatility produced by our two-factor model would then differ markedly depending on why today's volatility is high. If, for example, today's persistent volatility component is high and the transient component is not, then the forecast would be for continued high volatility. If, on the other hand, today's transient volatility component is high and the persistent volatility component is not, then the forecast would be for quick reversion of volatility to its mean. The ability to disentangle these effects is lost when one uses a reduced-form representation, which effectively attributes average persistence to all shocks.

${ }^{33}$ Multiple exchange rate volatility factors are also predicted by modern financial economic theory, as for example in Bansal (1997). 


\section{Concluding Remarks and Directions for Future Research}

The range has a long history in finance, from the stock charts in business newspapers to highbrow academics. We have clarified the properties of the log range as a volatility proxy, and we have used it to implement simple yet highly efficient maximum likelihood estimation of stochastic volatility models, facilitating our detailed examination of the volatility dynamics of five major U.S. dollar exchange rates. Our empirical results are sharp and surprising, strongly indicating two volatility factors operative in each exchange rate, with one reverting slowly to its mean and controlling volatility persistence, and one reverting quickly to its mean and controlling the volatility of volatility.

Our empirical work is built on a foundation of both theoretical and Monte Carlo analysis establishing that the log range is nearly Gaussian, much less noisy than popular alternative volatility proxies such as log absolute or squared returns, and robust to bid-ask bounce and related microstructure noise, unlike competitors such as realized volatility. Those properties of the range translate into a simple range-based Gaussian quasi-maximum likelihood estimator that is highly efficient, in small as well as large samples, and widely applicable for studying stochastic volatility dynamics in financial asset returns.

We look forward to pursuing future research in several directions. On the empirical side, more work is needed on the number, nature and determinants of the factors underlying stochastic volatility. It is striking that only a few years ago the possibility of multiple volatility factors was rarely, if ever, entertained, as one-factor models appeared adequate. Presently, however, it appears that a consensus is emerging for two-factor stochastic volatility dynamics, whether in equity or foreign exchange, despite the different natures of the assets and the different market 
microstructures. Much of the most relevant research has been done very recently; particularly noteworthy contributions include Jacquier, Polson, and Rossi (1994,1999), Gallant, Hsu, and Tauchen (1999), Jacquier and Polson (2000), and Chernov, Gallant, Ghysels, and Tauchen (2001), Barndorff-Nielsen and Shephard (2000, 2001) and Bollerslev and Zhou (2001). Lo and Wang (2000) provide interesting related evidence, finding two factors in equity trading volume.

Further empirical advances will require further financial econometric developments, including multivariate extensions of range-based volatility proxies and more thorough comparison of the range to another highly efficient volatility proxy that has received recent attention, namely realized volatility constructed from high-frequency intraday data. We have noted, for example, the intriguing robustness of the range to some common sources of microstructure noise, but a more extensive exploration and comparison of range-based volatility to realized volatility is needed, particularly as ongoing work such as that summarized in Andersen, Bollerslev, Diebold, and Labys (2000) develops methods of making realized volatility robust to microstructure noise. 


\section{References}

Alizadeh, Sassan 1998, Essays in Financial Econometrics, Ph.D. Dissertation, Department of Economics, University of Pennsylvania.

Andersen, Torben G., and Tim Bollerslev, 1997, Heterogeneous information arrivals and return volatility dynamics: Uncovering the long-run in high frequency returns, Journal of Finance 52, 975-1005.

Andersen, Torben G., and Tim Bollerslev, 1998, Answering the skeptics: Yes, standard volatility models do provide accurate forecasts, International Economic Review 39, 885-905.

Andersen, Torben G., Tim Bollerslev, Francis X. Diebold, and Heiko Ebens, 2001, The distribution of realized stock return volatility, Journal of Financial Economics 61, in press.

Andersen, Torben G., Tim Bollerslev, Francis X. Diebold, and Paul Labys, 2000, Great realizations, Risk 13, 105-108.

Andersen, Torben G., Tim Bollerslev, Francis X. Diebold, and Paul Labys, 2001, The distribution of realized exchange rate volatility, Journal of the American Statistical Association, 96, 42-55.

Andersen, Torben G., and Bent E. Sorensen (1997), GMM and QML asymptotic standard deviations in stochastic volatility models, Journal of Econometrics 76, 397-403.

Ball, Clifford A., and Walter N. Torous, 1984, The maximum likelihood estimation of security price volatility: Theory, evidence, and application to option pricing, Journal of Business 57, 97-112.

Bansal, Ravi (1997), An exploration of the forward premium puzzle in currency markets, Review of Financial Studies 10, 369-403.

Barndorff-Nielsen, Ole E., and Neil Shephard (2000), Econometric analysis of realized volatility and its use in estimating Lévy-based non-Gaussian OU type stochastic volatility models, Working paper, Nuffield College, Oxford.

Barndorff-Nielsen, Ole E., and Neil Shephard (2001), Non-Gaussian Ornstein-Uhlenbeck-based models and some of their uses in financial economics, Journal of the Royal Statistical Society B 63, in press.

Bates, David S., 1996, Jumps and stochastic volatility: Exchange rate processes implicit in Deutschemark options, Review of Financial Studies 9, 69-107. 
Beckers, Stan, 1983, Variance of security price returns based on high, low, and closing prices, Journal of Business 56, 97-112.

Black, Fischer, and Myron Scholes, 1973, The pricing of options and corporate liabilities, Journal of Political Economy 81, 637-654.

Bollerslev, Tim, Ray Y. Chou, and Kenneth F. Kroner, 1992, ARCH modeling in finance: A selective review of the theory and empirical evidence, Journal of Econometrics 52, 5-59.

Bollerslev, Tim, and Hao Zhou (2001), Estimating stochastic volatility diffusions using conditional moments of integrated volatility, Working Paper, Duke University.

Breidt, F. Jay, and A.L. Carriquiry, 1996, Improved quasi-maximum likelihood estimation for stochastic volatility models, in J.C. Lee, W.O. Johnson, and A. Zellner (eds.), Modelling and Prediction. New York: Springer Verlag, 228-247.

Brown, Stephen, 1990, Estimating volatility, in S. Figlewski, W. Silber, and M. Subrahmanyam (eds.), Financial Options. Homewood, IL.: Business One Irwin.

Chernov, Mikhail, and Eric Ghysels (2000), A study toward a unified approach to the joint estimation of objective and risk neutral measures for options valuation, Journal of Financial Economics 56, 407-458.

Chernov, Mikhail, Eric Ghysels, A. Ronald Gallant, and George E. Tauchen, 2001, Alternative models for stock price dynamics, Working Paper, Columbia University, University of North Carolina, and Duke University.

Danielsson, Jon, 1994, Stochastic volatility in asset prices: Estimation with simulated maximum likelihood, Journal of Econometrics 64, 375-400.

Doob, Joseph L., 1949, Heuristic approach to Kolmogorov-Simirnov theorems, Annals of Mathematical Statistics 20, 393-403.

Duffie, Darrell, Pan, Jun, and Kenneth Singleton, 2000, Transform analysis and asset pricing for affine jump-diffusions, Econometrica 68, 1343-1376.

Duffie, Darrell, and Kenneth Singleton, 1993, Simulated moments estimation of Markov models of asset prices, Econometrica 61, 929-952.

Edwards, Robert D., and John Magee, 1997, Technical Analysis of Stock Trends, New York: Amacom.

Engle, Robert F., and Gary G.J. Lee, 1999, A permanent and transitory model of stock return volatility, in R. Engle and H. White (eds.), Cointegration, Causality, and Forecasting: A Festschrift in Honor of Clive W.J. Granger, 475-497. Oxford: Oxford University Press. 
Engle, Robert F., and Victor K. Ng, 1993, Measuring and testing the impact of news on volatility, Journal of Finance 48, 1749-1801.

Feller, William, 1951, The asymptotic distribution of the range of sums of independent random variables, Annals of Mathematical Statistics 22, 427-432.

French, Kenneth R., G. William Schwert, and Robert F. Stambaugh, 1987, Expected stock returns and volatility, Journal of Financial Economics 19, 3-29.

Fridman, Moshe, and Lawrence Harris, 1998, A Maximum likelihood approach for non-Gaussian stochastic volatility models, Journal of Business and Economic Statistics 16, 284-291.

Galant, A. Ronald, David A. Hsieh, and George E. Tauchen, 1997, Estimation of stochastic volatility models with diagnostics, Journal of Econometrics 81, 159-192.

Gallant, A.Ronald, Chien T. Hsu, and George E. Tauchen, 1999, Using daily range data to calibrate volatility diffusions and extract the forward integrated variance, Review of Economics and Statistics 81, 617-631.

Garman, Mark B., and Michael J. Klass, 1980, On the estimation of price volatility from historical data, Journal of Business 53, 67-78.

Ghysels, Eric, Andrew Harvey, and Eric Renault, 1996, Stochastic volatility, in G.S. Maddala and C.R. Rao (eds.), Statistical Methods in Finance (Handbook of Statistics, Volume 14). Amsterdam: North-Holland.

Hamilton, James D., 1994, Time Series Analysis. Princeton: Princeton University Press.

Harvey, Andrew, Esther Ruiz, and Neil Shephard, 1994, Multivariate stochastic variance models, Review of Economic Studies 61, 247-264.

Harvey, Andrew, Esther Ruiz, and Neil Shephard, 1996, Estimation of an asymmetric stochastic volatility model for asset returns, Journal of Business and Economic Statistics 14, 429434.

Hasbrouck, Joel, 1996, Modeling market microstructure time series, in G.S. Maddala and C.R. Rao (eds.), Statistical Methods in Finance (Handbook of Statistics, Volume 14). Amsterdam: North-Holland, 647-692.

Hasbrouck, Joel, 1999, The dynamics of discrete bid and ask quotes, Journal of Finance 54, 2109-2142.

Heston, Steven L., 1993, A closed-form solution for options with stochastic volatility with applications to bond and currency options, Review of Financial Studies 6, 327-343. 
Hull, John C., and Alan D. White, 1987, The pricing of options on assets with stochastic volatilities, Journal of Finance 42, 381-400.

Jacquier, Eric, and Nicholas G. Polson, 2000, Stochastic volatility and other shocks in financial markets: an odds ratio analysis, Working Paper, Boston College and University of Chicago.

Jacquier, Eric, Nicholas G. Polson, and Peter E. Rossi, 1994, Bayesian analysis of stochastic volatility models, Journal of Business and Economic Statistics 12, 413-417.

Jacquier, Eric, Nicholas G. Polson, and Peter E. Rossi, 1999, Stochastic volatility: Univariate and multivariate extensions, Working Paper, Boston College and University of Chicago.

Jarrow, Robert A., 1998, Volatility. London: Risk Publications.

Karatzas, Ioannis, and Steven E. Shreve, 1991, Brownian Motion and Stochastic Calculus, New York: Springer Verlag.

Kim, Sangjoon, Neil Shephard, and Siddartha Chib, 1998, Stochastic volatility: Likelihood inference and comparison with ARCH models, Review of Economic Studies 65, 361-393.

Lo, A.W. and J. Wang (2000), "Trading Volume: Definitions, Data Analysis, and Implications of Portfolio Theory," Review of Financial Studies, 13, 257-300.

Merton, Robert C., 1969, Lifetime portfolio selection under uncertainty: The continuous-time case, Review of Economics and Statistics 51, 247-257.

Merton, Robert C., 1980, On estimating the expected return on the market: An exploratory investigation, Journal of Financial Economics 8, 323-361.

Nelson, Daniel B., 1991, Conditional heteroskedasticity in asset pricing: A new approach, Econometrica 59, 347-370.

Parkinson, Michael, 1980, The extreme value method for estimating the variance of the rate of return, Journal of Business 53, 61-65.

Rogers, L.C.G., and Stephen E. Satchell, 1991, Estimating variance from high, low and closing prices, Annals of Applied Probability 1, 504-512.

Ruiz, Esther, 1994, Quasi-maximum likelihood estimation of stochastic variance models, Journal of Econometrics 63, 289-306.

Sandmann, Gleb, and S.J. Koopman, 1998, Estimation of stochastic volatility models via Monte Carlo maximum likelihood, Journal of Econometrics 87, 271-301. 
Schwert, G. William, 1989, Why does stock market volatility change over time?, Journal of Finance 44, 1115-1153.

Schwert, G. William, 1990, Stock volatility and the crash of '87, Review of Financial Studies 3, 77-102.

Singleton, Kenneth, 1997, Estimation of affine asset pricing models using the empirical characteristic function, Working Paper, Stanford University.

Yang, Dennis, and Qiang Zhang, 2000, Drift-independent volatility estimation based on high, low, open and close prices, Journal of Business 73, 477-491. 


\section{Table I}

\section{Moments of Alternative Volatility Proxies}

We consider a driftless Brownian motion $x$, with origin $x_{0}=0$ and constant diffusion coefficient $\sigma$, over an interval of finite length $\tau$. The table shows the first four moments of two volatility proxies: the $\log$ absolute return $\ln \left|x_{\tau}\right|$ and the log range $\ln \left|\sup x_{t}-\inf x_{t}\right|$.

\begin{tabular}{lcccc}
\hline Volatility Proxy & Mean & $\begin{array}{l}\text { Standard } \\
\text { Deviation }\end{array}$ & Skewness & Kurtosis \\
\hline & & & & \\
Log Absolute Return & $-0.64+1 / 2 \ln \tau+\ln \sigma$ & 1.11 & -1.53 & 6.93 \\
Log Range & $0.43+1 / 2 \ln \tau+\ln \sigma$ & 0.29 & 0.17 & 2.80 \\
\hline
\end{tabular}




\section{Table II \\ Sampling Distributions of Estimators of the Parameters of the Stochastic Volatility Model, with $T=1000$ Observations}

We report statistics summarizing the sampling distribution of three estimators of the parameters and the latent volatilities of the stochastic volatility model:

$$
\begin{gathered}
s_{t}=s_{t-\Delta t}+\sigma_{i H} \varepsilon_{s t} \sqrt{\Delta t} \\
\ln \sigma_{(i+1) H}=\ln \bar{\sigma}+\rho_{H}\left(\ln \sigma_{i H}-\ln \bar{\sigma}\right)+\beta \varepsilon_{v i} \sqrt{H}
\end{gathered}
$$

$i H<t \leq(i+1) H$, where $\varepsilon_{s t}$ and $\varepsilon_{v i}$ are independent $\mathrm{N}[0,1]$ variates. We set $H=1 / 257$ and $\Delta t=H / N$, which corresponds to using daily data generated by $N$ trades per day. We consider $N=1000$ (A), $N=100$ (B), and $N=50(\mathrm{C})$. We set $\alpha=3.855, \ln \bar{\sigma}=-2.5$ and $\beta=0.75$, which implies a volatility process with daily autocorrelation of $\rho_{H}=0.985$, an annualized average volatility of 8.51 percent, and a coefficient of variation of 0.28. "QML with Absolute Return" denotes Gaussian quasimaximum likelihood estimation with the log absolute return as volatility proxy. "QML with Range" denotes Gaussian quasi-maximum likelihood estimation with the log range as volatility proxy. "Exact ML with Absolute Return" denotes a simulation-based estimator that maximizes the exact likelihood of log absolute returns. All results are based on 5000 replications. 
Table II A

Sampling Distribution with $T=1000$ Observations and $N=1000$ Trades

\begin{tabular}{|c|c|c|c|c|c|c|c|c|c|c|c|}
\hline & \multicolumn{3}{|c|}{ Parameter Estimates } & \multicolumn{4}{|c|}{ Prediction Errors with Estimated Parameters } & \multicolumn{4}{|c|}{ Prediction Errors with True Parameters } \\
\hline & $\rho=0.985$ & $\beta=0.750$ & $\ln \bar{\sigma}=-2.5$ & Mean & RMS & Mean $\%$ & RMS \% & Mean & RMS & Mean $\%$ & RMS \% \\
\hline & \multicolumn{11}{|c|}{ QML with Absolute Return } \\
\hline Mean & 0.946 & 1.078 & -2.498 & -0.0023 & 0.0185 & 0.026 & 0.218 & -0.0014 & 0.0167 & 0.023 & 0.197 \\
\hline RMSE & 0.142 & 1.175 & 0.101 & 0.0084 & 0.0053 & 0.096 & 0.046 & 0.0035 & 0.0040 & 0.039 & 0.025 \\
\hline $5 \%$ & 0.848 & 0.354 & -2.661 & -0.0172 & 0.0132 & -0.118 & 0.164 & -0.0075 & 0.0113 & -0.039 & 0.160 \\
\hline $25 \%$ & 0.964 & 0.629 & -2.564 & -0.0073 & 0.0149 & -0.042 & 0.186 & -0.0034 & 0.0139 & -0.004 & 0.179 \\
\hline $50 \%$ & 0.978 & 0.830 & -2.498 & -0.0013 & 0.0168 & 0.023 & 0.207 & -0.0012 & 0.0160 & 0.021 & 0.194 \\
\hline $75 \%$ & 0.987 & 1.111 & -2.429 & 0.0037 & 0.0205 & 0.088 & 0.237 & 0.0010 & 0.0188 & 0.048 & 0.211 \\
\hline \multirow[t]{2}{*}{$95 \%$} & 0.994 & 2.243 & -2.331 & 0.0093 & 0.0292 & 0.192 & 0.306 & 0.0039 & 0.0242 & 0.090 & 0.239 \\
\hline & \multicolumn{11}{|c|}{ QML with Range } \\
\hline Mean & 0.979 & 0.800 & -2.533 & -0.0012 & 0.0118 & 0.013 & 0.140 & -0.0027 & 0.0097 & -0.021 & 0.109 \\
\hline RMSE & 0.012 & 0.122 & 0.099 & 0.0079 & 0.0041 & 0.094 & 0.039 & 0.0013 & 0.0021 & 0.011 & 0.006 \\
\hline $5 \%$ & 0.961 & 0.633 & -2.682 & -0.0155 & 0.0084 & -0.130 & 0.104 & -0.0051 & 0.0067 & -0.039 & 0.099 \\
\hline $25 \%$ & 0.974 & 0.715 & -2.593 & -0.0059 & 0.0093 & -0.053 & 0.113 & -0.0035 & 0.0082 & -0.028 & 0.104 \\
\hline $50 \%$ & 0.981 & 0.790 & -2.534 & -0.0003 & 0.0104 & 0.008 & 0.127 & -0.0026 & 0.0094 & -0.021 & 0.109 \\
\hline $75 \%$ & 0.985 & 0.873 & -2.470 & 0.0045 & 0.0127 & 0.072 & 0.153 & -0.0017 & 0.0109 & -0.013 & 0.112 \\
\hline \multirow[t]{2}{*}{$95 \%$} & 0.990 & 0.988 & -2.384 & 0.0097 & 0.0200 & 0.172 & 0.222 & -0.0008 & 0.0133 & -0.002 & 0.118 \\
\hline & \multicolumn{11}{|c|}{ Exact ML with Absolute Return } \\
\hline Mean & 0.978 & 0.791 & -2.504 & -0.0020 & 0.0139 & 0.011 & 0.159 & -0.0011 & 0.0114 & 0.003 & 0.131 \\
\hline RMSE & 0.016 & 0.207 & 0.096 & 0.0083 & 0.0046 & 0.094 & 0.038 & 0.0020 & 0.0021 & 0.023 & 0.012 \\
\hline $5 \%$ & 0.953 & 0.491 & -2.660 & -0.0167 & 0.0098 & -0.134 & 0.120 & -0.0044 & 0.0083 & -0.035 & 0.113 \\
\hline $25 \%$ & 0.973 & 0.649 & -2.563 & -0.0067 & 0.0110 & -0.055 & 0.135 & -0.0024 & 0.0101 & -0.012 & 0.122 \\
\hline $50 \%$ & 0.981 & 0.777 & -2.505 & -0.0009 & 0.0124 & 0.007 & 0.149 & -0.0010 & 0.0112 & 0.003 & 0.130 \\
\hline $75 \%$ & 0.987 & 0.915 & -2.438 & 0.0041 & 0.0152 & 0.072 & 0.174 & 0.0002 & 0.0127 & 0.019 & 0.140 \\
\hline $95 \%$ & 0.992 & 1.145 & -2.346 & 0.0095 & 0.0230 & 0.170 & 0.238 & 0.0020 & 0.0152 & 0.040 & 0.152 \\
\hline
\end{tabular}




\section{Table II B}

\section{Sampling Distribution with $T=1000$ Observations and $N=100$ Trades}

\begin{tabular}{|c|c|c|c|c|c|c|c|c|c|c|c|}
\hline & \multicolumn{3}{|c|}{ Parameter Estimates } & \multicolumn{4}{|c|}{ Prediction Errors with Estimated Parameters } & \multicolumn{4}{|c|}{ Prediction Errors with True Parameters } \\
\hline & $\rho=0.985$ & $\beta=0.750$ & $\ln \bar{\sigma}=-2.5$ & Mean & RMS & Mean \% & RMS \% & Mean & RMS & Mean \% & RMS \% \\
\hline & \multicolumn{11}{|c|}{ QML with Range } \\
\hline Mean & 0.974 & 0.889 & -2.593 & -0.0012 & 0.0116 & 0.015 & 0.146 & -0.0066 & 0.0112 & -0.073 & 0.127 \\
\hline RMSE & 0.016 & 0.195 & 0.134 & 0.0079 & 0.0043 & 0.097 & 0.041 & 0.0021 & 0.0027 & 0.012 & 0.007 \\
\hline $5 \%$ & 0.951 & 0.676 & -2.749 & -0.0160 & 0.0082 & -0.138 & 0.107 & -0.0103 & 0.0074 & -0.092 & 0.115 \\
\hline $25 \%$ & 0.969 & 0.794 & -2.657 & -0.0054 & 0.0091 & -0.052 & 0.118 & -0.0078 & 0.0093 & -0.081 & 0.122 \\
\hline $95 \%$ & 0.989 & 1.125 & -2.434 & 0.0097 & 0.0206 & 0.185 & 0.236 & -0.0037 & 0.0165 & -0.052 & 0.139 \\
\hline
\end{tabular}

Table II C

Sampling Distribution with $T=1000$ Observations and $N=50$ Trades

\begin{tabular}{|c|c|c|c|c|c|c|c|c|c|c|c|}
\hline & \multicolumn{3}{|c|}{ Parameter Estimates } & \multicolumn{4}{|c|}{ Prediction Errors with Estimated Parameters } & \multicolumn{4}{|c|}{ Prediction Errors with True Parameters } \\
\hline & $\rho=0.985$ & $\beta=0.750$ & $\ln \bar{\sigma}=-2.5$ & Mean & RMS & Mean $\%$ & RMS \% & Mean & RMS & Mean $\%$ & RMS \% \\
\hline & \multicolumn{11}{|c|}{ QML with Range } \\
\hline Mean & 0.967 & 1.007 & -2.632 & -0.0011 & 0.0113 & 0.014 & 0.148 & -0.0087 & 0.0125 & -0.105 & 0.147 \\
\hline RMSE & 0.026 & 0.315 & 0.162 & 0.0073 & 0.0038 & 0.095 & 0.039 & 0.0023 & 0.0029 & 0.011 & 0.008 \\
\hline $5 \%$ & 0.933 & 0.742 & -2.785 & -0.0138 & 0.0082 & -0.130 & 0.111 & -0.0129 & 0.0085 & -0.124 & 0.134 \\
\hline $25 \%$ & 0.960 & 0.884 & -2.699 & -0.0054 & 0.0090 & -0.054 & 0.121 & -0.0102 & 0.0103 & -0.113 & 0.141 \\
\hline $95 \%$ & 0.987 & 1.330 & -2.482 & 0.0088 & 0.0183 & 0.176 & 0.227 & -0.0054 & 0.0176 & -0.087 & 0.161 \\
\hline
\end{tabular}




\section{Table III}

\section{Sampling Distribution with $\boldsymbol{T}=\mathbf{5 0 0}$ Observations}

We report statistics summarizing the sampling distribution of three estimators of the parameters and the latent volatilities of the stochastic volatility model:

$$
\begin{gathered}
s_{t}=s_{t-\Delta t}+\sigma_{i H} \varepsilon_{s t} \sqrt{\Delta t} \\
\ln \sigma_{(i+1) H}=\ln \bar{\sigma}+\rho_{H}\left(\ln \sigma_{i H}-\ln \bar{\sigma}\right)+\beta \varepsilon_{v i} \sqrt{H},
\end{gathered}
$$

$i H<t \leq(i+1) H$, where $\varepsilon_{s t}$ and $\varepsilon_{v i}$ are independent $\mathrm{N}[0,1]$ variates. We set $H=1 / 257$ and $\Delta t=H / N$, which corresponds to using daily data generated by $N$ trades per day. We consider $N=1000$ (A), $N=100$ (B), and $N=50(\mathrm{C})$. We set $\alpha=3.855, \ln \bar{\sigma}=-2.5$ and $\beta=0.75$, which implies a volatility process with daily autocorrelation of $\rho_{H}=0.985$, an annualized average volatility of 8.51 percent, and a coefficient of variation of 0.28. "QML with Absolute Return" denotes Gaussian quasimaximum likelihood estimation with the log absolute return as volatility proxy. "QML with Range" denotes Gaussian quasi-maximum likelihood estimation with the log range as volatility proxy. "Exact ML with Absolute Return" denotes a simulation-based estimator that maximizes the exact likelihood of log absolute returns. All results are based on 5000 replications. 
Table III A

Sampling Distribution with $T=500$ Observations and $N=1000$ Trades

\begin{tabular}{|c|c|c|c|c|c|c|c|}
\hline & \multicolumn{3}{|c|}{ Parameter Estimates } & \multicolumn{4}{|c|}{ Prediction Errors with Estimated Parameters } \\
\hline & $\rho=0.985$ & $\beta=0.750$ & $\ln \bar{\sigma}=-2.5$ & Mean & RMS & Mean $\%$ & RMS \% \\
\hline & \multicolumn{7}{|c|}{ QML with Absolute Return } \\
\hline Mean & 0.862 & 1.604 & -2.496 & -0.0033 & 0.0200 & 0.028 & 0.233 \\
\hline RMSE & 0.288 & 2.153 & 0.138 & 0.0121 & 0.0084 & 0.130 & 0.067 \\
\hline $5 \%$ & 0.095 & 0.295 & -2.712 & -0.0259 & 0.0125 & -0.172 & 0.159 \\
\hline $25 \%$ & 0.917 & 0.629 & -2.590 & -0.0090 & 0.0148 & -0.061 & 0.186 \\
\hline $50 \%$ & 0.969 & 0.918 & -2.497 & -0.0007 & 0.0172 & 0.023 & 0.217 \\
\hline $75 \%$ & 0.984 & 1.523 & -2.403 & 0.0053 & 0.0220 & 0.113 & 0.262 \\
\hline \multirow[t]{2}{*}{$95 \%$} & 0.993 & 6.293 & -2.261 & 0.0120 & 0.0372 & 0.249 & 0.367 \\
\hline & \multicolumn{7}{|c|}{ QML with Range } \\
\hline Mean & 0.972 & 0.817 & -2.531 & -0.0023 & 0.0135 & 0.014 & 0.157 \\
\hline RMSE & 0.023 & 0.180 & 0.129 & 0.0113 & 0.0071 & 0.125 & 0.058 \\
\hline $5 \%$ & 0.936 & 0.554 & -2.731 & -0.0242 & 0.0082 & -0.185 & 0.102 \\
\hline $25 \%$ & 0.967 & 0.701 & -2.623 & -0.0080 & 0.0093 & -0.071 & 0.116 \\
\hline $50 \%$ & 0.977 & 0.804 & -2.533 & -0.0001 & 0.0110 & 0.008 & 0.138 \\
\hline $75 \%$ & 0.985 & 0.918 & -2.448 & 0.0060 & 0.0145 & 0.100 & 0.181 \\
\hline \multirow[t]{2}{*}{$95 \%$} & 0.991 & 1.092 & -2.318 & 0.0116 & 0.0283 & 0.228 & 0.273 \\
\hline & \multicolumn{7}{|c|}{ Exact ML with Absolute Return } \\
\hline Mean & 0.964 & 0.863 & -2.504 & -0.0036 & 0.0157 & 0.007 & 0.176 \\
\hline RMSE & 0.052 & 0.407 & 0.132 & 0.0120 & 0.0079 & 0.126 & 0.056 \\
\hline $5 \%$ & 0.898 & 0.399 & -2.714 & -0.0260 & 0.0093 & -0.190 & 0.117 \\
\hline $25 \%$ & 0.960 & 0.634 & -2.591 & -0.0091 & 0.0110 & -0.079 & 0.137 \\
\hline $50 \%$ & 0.977 & 0.805 & -2.507 & -0.0013 & 0.0130 & -0.001 & 0.161 \\
\hline $75 \%$ & 0.985 & 1.029 & -2.417 & 0.0049 & 0.0169 & 0.090 & 0.200 \\
\hline $95 \%$ & 0.993 & 1.475 & -2.283 & 0.0115 & 0.0323 & 0.225 & 0.292 \\
\hline
\end{tabular}


Table III B

Sampling Distribution with $T=500$ Observations and $N=100$ Trades

\begin{tabular}{|c|c|c|c|c|c|c|c|}
\hline & & \multicolumn{2}{|c|}{ Parameter Estimates } & \multicolumn{4}{|c|}{ Prediction Errors with Estimated Parameters } \\
\hline & $\rho=0.985$ & $\beta=0.750$ & $\ln \bar{\sigma}=-2.5$ & Mean & RMS & Mean \% & RMS \% \\
\hline & \multicolumn{7}{|c|}{ QML with Range } \\
\hline Mean & 0.964 & 0.936 & -2.590 & -0.0024 & 0.0135 & 0.016 & 0.167 \\
\hline RMSE & 0.039 & 0.294 & 0.162 & 0.0114 & 0.0071 & 0.136 & 0.065 \\
\hline $5 \%$ & 0.915 & 0.630 & -2.810 & -0.0247 & 0.0081 & -0.193 & 0.107 \\
\hline $25 \%$ & 0.956 & 0.784 & -2.682 & -0.0084 & 0.0094 & -0.080 & 0.122 \\
\hline $50 \%$ & 0.971 & 0.911 & -2.593 & -0.0002 & 0.0109 & 0.009 & 0.147 \\
\hline $75 \%$ & 0.981 & 1.046 & -2.499 & 0.0058 & 0.0146 & 0.102 & 0.193 \\
\hline $95 \%$ & 0.990 & 1.326 & -2.365 & 0.0118 & 0.0291 & 0.251 & 0.295 \\
\hline
\end{tabular}

Table III C Sampling Distribution with $T=500$ Observations and $N=50$ Trades

\begin{tabular}{|c|c|c|c|c|c|c|c|}
\hline & \multicolumn{3}{|c|}{ Parameter Estimates } & \multicolumn{4}{|c|}{$\begin{array}{l}\text { Prediction Errors with Estimated Parameters } \\
\end{array}$} \\
\hline & $\rho=0.985$ & $\beta=0.750$ & $\ln \bar{\sigma}=-2.5$ & Mean & RMS & Mean \% & RMS \% \\
\hline & \multicolumn{7}{|c|}{ QML with Range } \\
\hline Mean & 0.949 & 1.093 & -2.628 & -0.0021 & 0.0127 & 0.013 & 0.165 \\
\hline RMSE & 0.070 & 0.490 & 0.178 & 0.0099 & 0.0059 & 0.126 & 0.058 \\
\hline $5 \%$ & 0.873 & 0.681 & -2.833 & -0.0207 & 0.0079 & -0.177 & 0.110 \\
\hline $25 \%$ & 0.944 & 0.873 & -2.711 & -0.0073 & 0.0093 & -0.073 & 0.124 \\
\hline $50 \%$ & 0.964 & 1.033 & -2.628 & -0.0004 & 0.0107 & 0.007 & 0.146 \\
\hline $75 \%$ & 0.977 & 1.231 & -2.546 & 0.0052 & 0.0138 & 0.092 & 0.187 \\
\hline $95 \%$ & 0.988 & 1.652 & -2.429 & 0.0106 & 0.0248 & 0.222 & 0.271 \\
\hline
\end{tabular}




\section{Table IV \\ Sampling Distributions \\ Realized and Range-Based Volatility Estimates}

We simulate one day of five-minute log prices (289 observations) from the Gaussian logarithmic random walk, $s_{t}=s_{t-1}+u_{t}$, with $u_{t} \sim \mathrm{NID}\left[0, \sigma_{u}^{2}\right]$. Let the bid price be $B_{t}=$ floor $\left[S_{t}\right.$-ticksize $]$, and let the ask price be $A_{t}=$ ceiling $\left[S_{t}+\operatorname{ticksize}\right]$, where $S_{t}=\exp \left(s_{t}\right)$ is the true price. We then take the observed price as $S_{t}^{o b s}=B_{t} q_{t}+A_{t}\left(1-q_{t}\right)$, where $q_{t}=$ Bernoulli[1/2]. Hence the observed price fluctuates randomly between the bid and the ask. We take $S_{0}=\$ 25$, ticksize $=\$ 1 / 16$, and $\sigma_{u}=0.0011$, which implies a fixed daily return volatility of 1.87 percent, and an annualized return volatility of thirty percent, assuming 250 trading days per year. For each day's data we calculate both realized and range-based volatility estimates (see text for details), based upon both true and observed returns, using a variety of underlying return intervals (5-minute, 10-minute, 20-minute, 40-minute, 1 hour and 20 minutes, 3 hour, 6 hour, and 12 hour). We repeat this 100,000 times, and we report moments of the corresponding distributions below, all of which are expressed as percentages.

\begin{tabular}{|c|c|c|c|c|c|c|}
\hline \multirow{2}{*}{$\begin{array}{l}\text { Return } \\
\text { Interval }\end{array}$} & \multicolumn{3}{|c|}{ Realized Volatility } & \multicolumn{3}{|c|}{ Range-Based Volatility } \\
\hline & Mean & Std & RMSE & Mean & Std & RMSE \\
\hline & \multicolumn{6}{|c|}{ True Returns } \\
\hline $5-\min$ & 1.87 & 0.08 & 0.08 & 1.71 & 0.53 & 0.55 \\
\hline $10-\min$ & 1.86 & 0.11 & 0.11 & 1.68 & 0.53 & 0.56 \\
\hline 20-min & 1.86 & 0.16 & 0.16 & 1.63 & 0.53 & 0.58 \\
\hline 40-min & 1.85 & 0.22 & 0.22 & 1.56 & 0.53 & 0.61 \\
\hline $1 \mathrm{hr} 20 \mathrm{~min}$ & 1.84 & 0.31 & 0.31 & 1.45 & 0.53 & 0.67 \\
\hline $3 \mathrm{hr}$ & 1.81 & 0.46 & 0.46 & 1.27 & 0.52 & 0.79 \\
\hline $6 \mathrm{hr}$ & 1.80 & 0.64 & 0.65 & 1.02 & 0.51 & 0.99 \\
\hline \multirow[t]{2}{*}{$12 \mathrm{hr}$} & 1.79 & 0.87 & 0.89 & 0.63 & 0.48 & 1.32 \\
\hline & \multicolumn{6}{|c|}{ Observed Returns } \\
\hline $5-\min$ & 9.35 & 0.32 & 7.49 & 2.11 & 0.53 & 0.59 \\
\hline 10-min & 6.74 & 0.32 & 4.88 & 2.06 & 0.53 & 0.57 \\
\hline 20-min & 4.94 & 0.34 & 3.09 & 1.98 & 0.53 & 0.54 \\
\hline 40-min & 3.72 & 0.39 & 1.89 & 1.87 & 0.53 & 0.53 \\
\hline $1 \mathrm{hr} 20 \mathrm{~min}$ & 2.92 & 0.45 & 1.15 & 1.71 & 0.53 & 0.55 \\
\hline $3 \mathrm{hr}$ & 2.34 & 0.58 & 0.75 & 1.44 & 0.53 & 0.68 \\
\hline $6 \mathrm{hr}$ & 2.03 & 0.73 & 0.75 & 1.13 & 0.54 & 0.91 \\
\hline $12 \mathrm{hr}$ & 1.79 & 0.93 & 0.94 & 0.68 & 0.52 & 1.29 \\
\hline
\end{tabular}


Table V

Distributions and Dynamics of Volatility Proxies

for Five Dollar Exchange Rates

We report statistics summarizing both the unconditional moments and the autocorrelations of two volatility proxies for five dollar exchange rates, measured daily from January 1, 1978 through December 31, 1998 (5284 observations). The underlying data used to compute the log absolute return and the log range are daily high, low, and settlement prices of front-month futures contracts traded on the International Monetary Market.

\begin{tabular}{|c|c|c|c|c|c|c|c|c|c|}
\hline \multirow[b]{2}{*}{ Volatility Proxy } & \multicolumn{4}{|c|}{ Unconditional Moments } & \multicolumn{5}{|c|}{ Autocorrelations } \\
\hline & Mean & Std Dev & Skew & Kurt & $1 \mathrm{st}$ & 2nd & 5 th & 10th & 20th \\
\hline & \multicolumn{9}{|c|}{ British Pound } \\
\hline Absolute Return & -5.82 & 1.19 & -0.86 & 3.64 & 0.09 & 0.06 & 0.10 & 0.07 & 0.05 \\
\hline \multirow[t]{2}{*}{ Range } & -4.87 & 0.53 & 0.09 & 3.10 & 0.39 & 0.33 & 0.30 & 0.27 & 0.22 \\
\hline & \multicolumn{9}{|c|}{ Canadian Dollar } \\
\hline Absolute Return & -6.67 & 1.10 & -0.62 & 2.89 & 0.11 & 0.09 & 0.12 & 0.08 & 0.05 \\
\hline \multirow[t]{2}{*}{ Range } & -5.70 & 0.53 & -0.02 & 3.39 & 0.49 & 0.46 & 0.41 & 0.35 & 0.31 \\
\hline & \multicolumn{9}{|c|}{ Deutsche Mark } \\
\hline Absolute Return & -5.77 & 1.17 & -0.88 & 3.62 & 0.06 & 0.06 & 0.09 & 0.07 & 0.05 \\
\hline \multirow[t]{2}{*}{ Range } & -4.83 & 0.52 & -0.07 & 3.12 & 0.40 & 0.37 & 0.35 & 0.30 & 0.23 \\
\hline & \multicolumn{9}{|c|}{ Japanese Yen } \\
\hline Absolute Return & -5.77 & 1.17 & -0.88 & 3.62 & 0.10 & 0.05 & 0.08 & 0.07 & 0.07 \\
\hline \multirow[t]{2}{*}{ Range } & -4.88 & 0.58 & 0.03 & 3.19 & 0.41 & 0.34 & 0.32 & 0.26 & 0.20 \\
\hline & \multicolumn{9}{|c|}{ Swiss Franc } \\
\hline Absolute Return & -5.60 & 1.16 & -0.95 & 3.77 & 0.05 & 0.02 & 0.06 & 0.05 & 0.04 \\
\hline Range & -4.67 & 0.48 & 0.04 & 3.13 & 0.32 & 0.29 & 0.30 & 0.25 & 0.19 \\
\hline
\end{tabular}




\section{Table VI \\ Quasi-Maximum Likelihood Estimates of \\ One-Factor and Two-Factor Stochastic Volatility Models \\ for Five Dollar Exchange Rates}

We report estimates of one-factor and two-factor stochastic volatility models fit to five dollar exchange rates, using daily data from January 1, 1978 through December 31, 1998. Asymptotic standard errors appear in parentheses. See text for model descriptions.

\begin{tabular}{|c|c|c|c|c|c|c|c|c|}
\hline \multirow[b]{2}{*}{ Volatility Proxy } & \multicolumn{3}{|c|}{ One Factor Model } & \multicolumn{5}{|c|}{ Two-Factor Model } \\
\hline & $\ln \bar{\sigma}$ & $\rho$ & $\beta$ & $\ln \bar{\sigma}$ & $\rho_{1}$ & $\beta_{1}$ & $\rho_{2}$ & $\beta_{2}$ \\
\hline & \multicolumn{8}{|c|}{ British Pound } \\
\hline Absolute Return & $\begin{array}{l}-2.42 \\
(0.06)\end{array}$ & $\begin{array}{c}0.99 \\
(0.01)\end{array}$ & $\begin{array}{c}0.91 \\
(0.18)\end{array}$ & $\begin{array}{l}-2.42 \\
(0.08)\end{array}$ & $\begin{array}{c}0.99 \\
(0.00)\end{array}$ & $\begin{array}{c}0.60 \\
(0.12)\end{array}$ & $\begin{array}{c}0.06 \\
(0.07)\end{array}$ & $\begin{array}{c}7.44 \\
(0.51)\end{array}$ \\
\hline \multirow[t]{2}{*}{ Range } & $\begin{array}{l}-2.51 \\
(0.01)\end{array}$ & $\begin{array}{c}0.65 \\
(0.02)\end{array}$ & $\begin{array}{c}5.33 \\
(0.12)\end{array}$ & $\begin{array}{l}-2.50 \\
(0.04)\end{array}$ & $\begin{array}{c}0.98 \\
(0.00)\end{array}$ & $\begin{array}{c}0.94 \\
(0.09)\end{array}$ & $\begin{array}{c}0.19 \\
(0.03)\end{array}$ & $\begin{array}{c}5.14 \\
(0.10)\end{array}$ \\
\hline & \multicolumn{8}{|c|}{ Canadian Dollar } \\
\hline Absolute Return & $\begin{array}{l}-3.29 \\
(0.06)\end{array}$ & $\begin{array}{c}0.98 \\
(0.01)\end{array}$ & $\begin{array}{c}1.12 \\
(0.16)\end{array}$ & $\begin{array}{l}-3.29 \\
(0.06)\end{array}$ & $\begin{array}{c}0.98 \\
(0.00)\end{array}$ & $\begin{array}{c}1.03 \\
(0.15)\end{array}$ & $\begin{array}{c}0.24 \\
(0.39)\end{array}$ & $\begin{array}{c}3.26 \\
(0.93)\end{array}$ \\
\hline \multirow[t]{2}{*}{ Range } & $\begin{array}{l}-3.34 \\
(0.02)\end{array}$ & $\begin{array}{c}0.85 \\
(0.01)\end{array}$ & $\begin{array}{c}3.69 \\
(0.14)\end{array}$ & $\begin{array}{l}-3.34 \\
(0.05)\end{array}$ & $\begin{array}{c}0.98 \\
(0.00)\end{array}$ & $\begin{array}{c}1.20 \\
(0.10)\end{array}$ & $\begin{array}{c}0.16 \\
(0.04)\end{array}$ & $\begin{array}{c}4.26 \\
(0.11)\end{array}$ \\
\hline & \multicolumn{8}{|c|}{ Deutsche Mark } \\
\hline Absolute Return & $\begin{array}{l}-2.38 \\
(0.04)\end{array}$ & $\begin{array}{c}0.97 \\
(0.01)\end{array}$ & $\begin{array}{c}1.37 \\
(0.25)\end{array}$ & $\begin{array}{l}-2.38 \\
(0.05)\end{array}$ & $\begin{array}{c}0.98 \\
(0.01)\end{array}$ & $\begin{array}{c}1.07 \\
(0.21)\end{array}$ & $\begin{array}{l}-0.11 \\
(0.16)\end{array}$ & $\begin{array}{c}6.57 \\
(0.61)\end{array}$ \\
\hline \multirow[t]{2}{*}{ Range } & $\begin{array}{l}-2.47 \\
(0.02)\end{array}$ & $\begin{array}{c}0.72 \\
(0.02)\end{array}$ & $\begin{array}{c}4.77 \\
(0.14)\end{array}$ & $\begin{array}{l}-2.47 \\
(0.04)\end{array}$ & $\begin{array}{c}0.97 \\
(0.01)\end{array}$ & $\begin{array}{c}1.23 \\
(0.09)\end{array}$ & $\begin{array}{c}0.05 \\
(0.04)\end{array}$ & $\begin{array}{c}4.64 \\
(0.11)\end{array}$ \\
\hline & \multicolumn{8}{|c|}{ Japanese Yen } \\
\hline Absolute Return & $\begin{array}{l}-2.37 \\
(0.04)\end{array}$ & $\begin{array}{c}0.97 \\
(0.01)\end{array}$ & $\begin{array}{c}1.47 \\
(0.28)\end{array}$ & $\begin{array}{l}-2.38 \\
(0.05)\end{array}$ & $\begin{array}{c}0.98 \\
(0.01)\end{array}$ & $\begin{array}{c}0.94 \\
(0.21)\end{array}$ & $\begin{array}{c}0.17 \\
(0.10)\end{array}$ & $\begin{array}{c}7.31 \\
(0.53)\end{array}$ \\
\hline \multirow[t]{2}{*}{ Range } & $\begin{array}{l}-2.53 \\
(0.02)\end{array}$ & $\begin{array}{c}0.62 \\
(0.02)\end{array}$ & $\begin{array}{c}6.20 \\
(0.12)\end{array}$ & $\begin{array}{l}-2.53 \\
(0.04)\end{array}$ & $\begin{array}{c}0.97 \\
(0.01)\end{array}$ & $\begin{array}{c}1.43 \\
(0.13)\end{array}$ & $\begin{array}{c}0.15 \\
(0.03)\end{array}$ & $\begin{array}{c}5.68 \\
(0.12)\end{array}$ \\
\hline & \multicolumn{8}{|c|}{ Swiss Franc } \\
\hline Absolute Return & $\begin{array}{l}-2.22 \\
(0.04)\end{array}$ & $\begin{array}{c}0.98 \\
(0.10)\end{array}$ & $\begin{array}{c}0.74 \\
(0.15)\end{array}$ & $\begin{array}{l}-2.22 \\
(0.05)\end{array}$ & $\begin{array}{c}0.99 \\
(0.00)\end{array}$ & $\begin{array}{c}0.59 \\
(0.13)\end{array}$ & $\begin{array}{c}0.02 \\
(0.02)\end{array}$ & $\begin{array}{c}6.29 \\
(0.58)\end{array}$ \\
\hline Range & $\begin{array}{l}-2.32 \\
(0.01)\end{array}$ & $\begin{array}{c}0.63 \\
(0.02)\end{array}$ & $\begin{array}{c}4.78 \\
(0.13)\end{array}$ & $\begin{array}{l}-2.32 \\
(0.03)\end{array}$ & $\begin{array}{c}0.97 \\
(0.01)\end{array}$ & $\begin{array}{c}1.05 \\
(0.08)\end{array}$ & $\begin{array}{c}0.03 \\
(0.03)\end{array}$ & $\begin{array}{c}4.50 \\
(0.11)\end{array}$ \\
\hline
\end{tabular}




\section{Table VII \\ Residual Diagnostics for \\ One-Factor Stochastic Volatility Models \\ for Five Dollar Exchange Rates}

We report statistics summarizing both the unconditional moments and the autocorrelations of measurement equation residuals from one-factor stochastic volatility models fit to five dollar exchange rates, using daily data from January 1, 1978 through December 31, 1998.

\begin{tabular}{|c|c|c|c|c|c|c|c|c|}
\hline \multirow[b]{2}{*}{ Volatility Proxy } & \multicolumn{3}{|c|}{ Unconditional Moments } & \multicolumn{5}{|c|}{ Autocorrelations } \\
\hline & Std Dev & Skew & Kurt & $1 \mathrm{st}$ & $2 \mathrm{nd}$ & 5 th & 10th & 20th \\
\hline & \multicolumn{8}{|c|}{ British Pound } \\
\hline Absolute Return & 1.17 & -1.29 & 5.87 & -0.00 & -0.02 & 0.02 & -0.00 & -0.02 \\
\hline \multirow[t]{2}{*}{ Range } & 0.30 & 0.15 & 3.06 & 0.18 & 0.20 & 0.22 & 0.20 & 0.16 \\
\hline & \multicolumn{8}{|c|}{ Canadian Dollar } \\
\hline Absolute Return & 1.10 & -1.19 & 5.46 & 0.10 & -0.01 & 0.02 & -0.02 & -0.03 \\
\hline \multirow[t]{2}{*}{ Range } & 0.26 & 0.17 & 3.25 & -0.02 & 0.07 & 0.12 & -0.10 & 0.09 \\
\hline & \multicolumn{8}{|c|}{ Deutsche Mark } \\
\hline Absolute Return & 1.16 & -1.46 & 7.53 & -0.03 & -0.02 & 0.02 & 0.00 & 0.00 \\
\hline \multirow[t]{2}{*}{ Range } & 0.28 & 0.06 & 3.07 & 0.10 & 0.16 & 0.21 & 0.18 & 0.15 \\
\hline & \multicolumn{8}{|c|}{ Japanese Yen } \\
\hline Absolute Return & 1.14 & -1.08 & 4.67 & 0.02 & -0.03 & 0.01 & 0.12 & 0.03 \\
\hline \multirow[t]{2}{*}{ Range } & 0.32 & 0.09 & 3.15 & 0.23 & 0.23 & 0.25 & 0.20 & 0.16 \\
\hline & \multicolumn{8}{|c|}{ Swiss Franc } \\
\hline Absolute Return & 1.15 & -1.25 & 5.59 & -0.00 & -0.04 & 0.01 & 0.01 & -0.00 \\
\hline Range & 0.19 & 0.12 & 3.08 & 0.12 & 0.16 & 0.21 & 0.18 & 0.15 \\
\hline
\end{tabular}




\section{Table VIII \\ Residual Diagnostics for \\ Two-Factor Stochastic Volatility Models \\ for Five Dollar Exchange Rates}

We report statistics summarizing both the unconditional moments and the autocorrelations of measurement equation residuals from two-factor stochastic volatility models fit to five dollar exchange rates, using daily data from January 1, 1978 through December 31, 1998.

\begin{tabular}{|c|c|c|c|c|c|c|c|c|}
\hline \multirow[b]{2}{*}{ Volatility Proxy } & \multicolumn{3}{|c|}{ Unconditional Moments } & \multicolumn{5}{|c|}{ Autocorrelations } \\
\hline & Std Dev & Skew & Kurt & $1 \mathrm{st}$ & $2 \mathrm{nd}$ & 5 th & 10th & 20th \\
\hline & \multicolumn{8}{|c|}{ British Pound } \\
\hline Absolute Return & 1.18 & -1.26 & 5.72 & 0.01 & -0.01 & 0.03 & 0.00 & -0.01 \\
\hline \multirow[t]{2}{*}{ Range } & 0.37 & 0.24 & 3.17 & 0.11 & 0.03 & 0.02 & 0.02 & 0.01 \\
\hline & \multicolumn{8}{|c|}{ Canadian Dollar } \\
\hline Absolute Return & 1.09 & -1.17 & 5.39 & 0.01 & -0.01 & 0.02 & -0.01 & -0.03 \\
\hline \multirow[t]{2}{*}{ Range } & 0.33 & 0.23 & 3.38 & 0.07 & 0.05 & 0.01 & -0.01 & 0.01 \\
\hline & \multicolumn{8}{|c|}{ Deutsche Mark } \\
\hline Absolute Return & 1.17 & -1.28 & 5.88 & -0.02 & -0.01 & 0.03 & 0.01 & 0.00 \\
\hline \multirow[t]{2}{*}{ Range } & 0.37 & 0.19 & 3.09 & 0.04 & 0.00 & 0.02 & 0.02 & 0.02 \\
\hline & \multicolumn{8}{|c|}{ Japanese Yen } \\
\hline Absolute Return & 1.16 & -1.34 & 6.72 & 0.03 & -0.01 & 0.02 & 0.02 & 0.02 \\
\hline \multirow[t]{2}{*}{ Range } & 0.39 & 0.26 & 3.27 & 0.09 & 0.01 & 0.03 & 0.01 & 0.02 \\
\hline & \multicolumn{8}{|c|}{ Swiss Franc } \\
\hline Absolute Return & 1.16 & -1.30 & 5.91 & 0.01 & -0.03 & 0.02 & 0.01 & 0.00 \\
\hline Range & 0.37 & 0.27 & 3.17 & 0.02 & -0.01 & 0.03 & 0.02 & 0.03 \\
\hline
\end{tabular}


Figure 1a

Distribution of Log Absolute Return

We consider a driftless Brownian motion, with zero origin and unit diffusion coefficient, over an interval of unit length. We plot the distribution of the log absolute return, with the best-fitting normal distribution superimposed for visual reference.

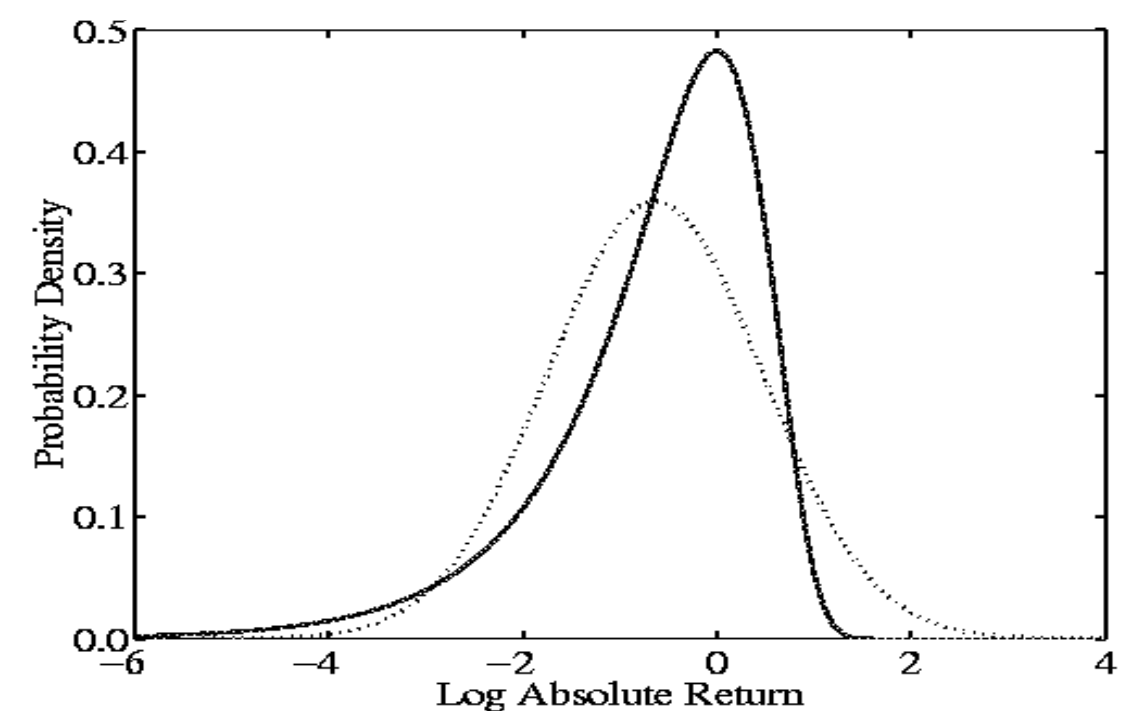

Figure 1b

Distribution of Log Range

We consider a driftless Brownian motion, with zero origin and unit diffusion coefficient, over an interval of unit length. We plot the distribution of the log range, with the best-fitting normal distribution superimposed for visual reference.

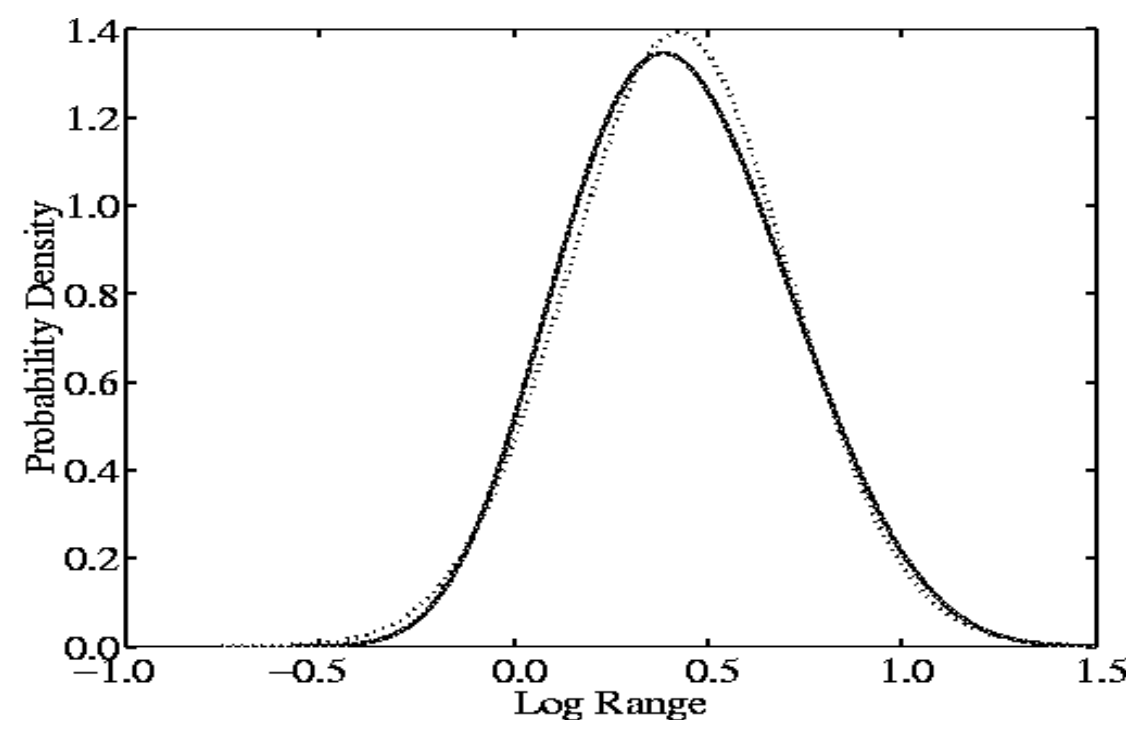




\section{Figure 2 \\ True and Observed Prices Simulated One-Day Sample Path}

We simulate one day of five-minute log prices (289 observations) from the Gaussian logarithmic random walk, $s_{t}=s_{t-1}+u_{t}$, with $u_{t} \sim \operatorname{NID}\left[0, \sigma_{u}^{2}\right]$. Let the bid price be $B_{t}=$ floor $\left[S_{t}\right.$-ticksize $]$, and let the ask price be $A_{t}=$ ceiling $\left[S_{t}+\right.$ ticksize $]$, where $S_{t}=\exp \left(s_{t}\right)$ is the true price. We then take the observed price as $S_{t}^{o b s}=B_{t} q_{t}+A_{t}\left(1-q_{t}\right)$, where $q_{t}=$ Bernoulli[1/2]. Hence the observed price fluctuates randomly between the bid and the ask. We take $S_{0}=\$ 25$, ticksize $=\$ 1 / 16$, and $\sigma_{u}=0.0011$, which implies an annualized thirty percent return volatility, assuming 250 trading days per year.

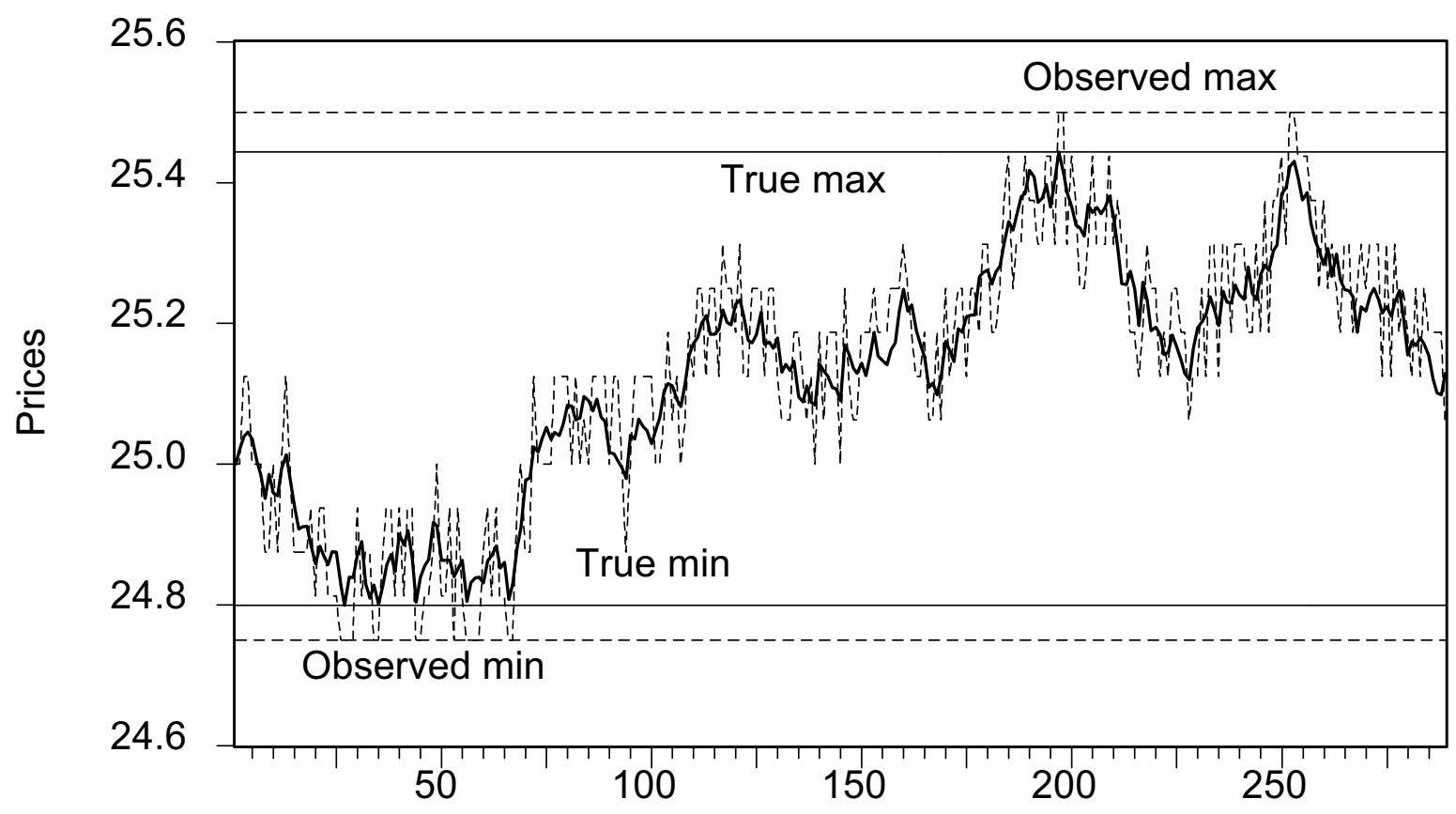

Time 
Figure 3

\section{Monte Carlo Distributions of Parameter Estimates}

We show the sampling distributions of three estimators of the parameters and the latent volatilities of the stochastic volatility model:

$$
\begin{gathered}
s_{t}=s_{t-\Delta t}+\sigma_{i H} \varepsilon_{s t} \sqrt{\Delta t} \\
\ln \sigma_{(i+1) H}=\ln \bar{\sigma}+\rho_{H}\left(\ln \sigma_{i H}-\ln \bar{\sigma}\right)+\beta \varepsilon_{v i} \sqrt{H},
\end{gathered}
$$

$i H<t \leq(i+1) H$, where $\varepsilon_{s t}$ and $\varepsilon_{v i}$ are independent $\mathrm{N}[0,1]$ variates. We set $H=1 / 257$ and $\Delta t=H / N$, which corresponds to using daily data generated by $N$ trades per day. We set $\alpha=3.855, \ln \bar{\sigma}=-2.5$ and $\sigma_{v}=0.75$, which implies a volatility process with daily autocorrelation of $\rho_{H}=0.985$, an annualized average volatility of 8.51 percent, and a coefficient of variation of 0.28. "QML with Absolute Return" denotes the Gaussian quasi-maximum likelihood estimator with the log absolute return as volatility proxy. "QML with Range" denotes the Gaussian quasi-maximum likelihood estimator with the log range as volatility proxy. "Exact ML with Absolute Return" denotes a simulation based estimator that maximizes the exact likelihood of log absolute returns. All results are based on 5000 Monte Carlo replications, a sample size of $T=1000$, and $N=1000$ trades per day. Reading across the rows, we show the sampling distributions of the estimators of $\rho, \beta, \log \bar{\sigma}$, and $E\left[\left(\hat{\sigma}_{t}-\sigma_{t}\right)^{2}\right]^{1 / 2}$. The two vertical lines in the second plot of the first row mark the range of the same plots in the second and third row.

QML with Absolute Return
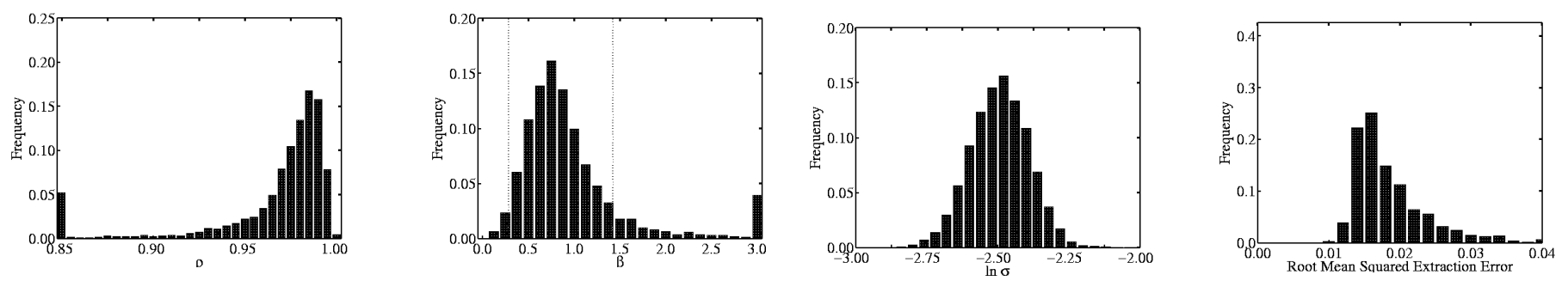

QML with Range
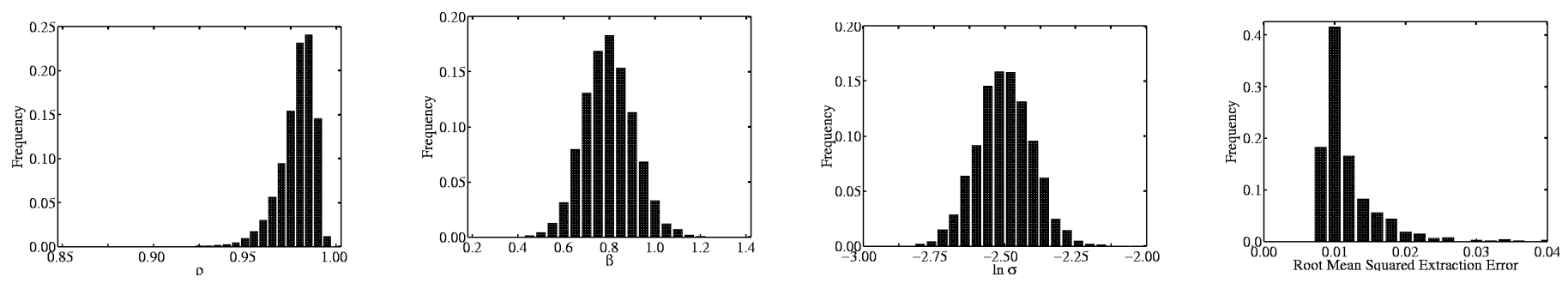

Exact ML with Absolute Return
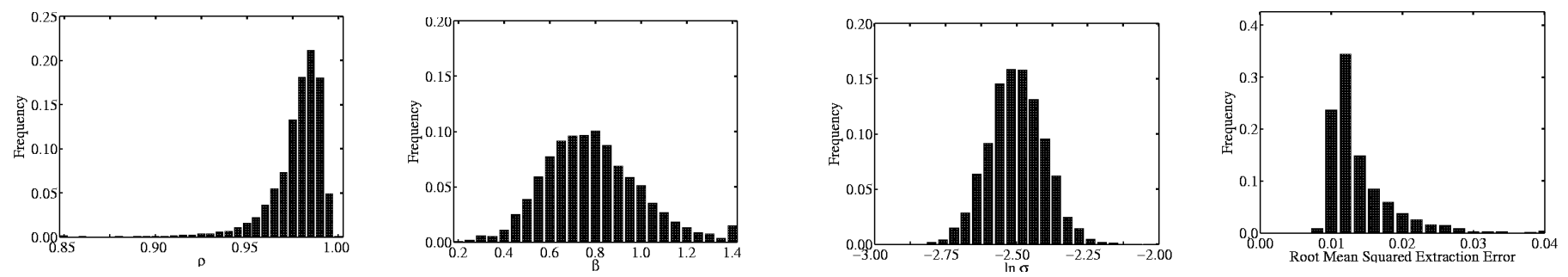


\section{Figure 4}

\section{Distributions of Realized Volatility and Range-Based Volatility Based on True Underlying Prices}

We simulate one day of five-minute log prices (289 observations) from the Gaussian logarithmic random walk, $s_{t}=s_{t-1}+u_{t}$, with $u_{t} \sim \mathrm{NID}\left[0, \sigma_{u}^{2}\right]$. Let the bid price be $B_{t}=$ floor $\left[S_{t}-\right.$ ticksize $]$, and let the ask price be $A_{t}=$ ceiling $\left[S_{t}+\right.$ ticksize $]$, where $S_{t}=\exp \left(s_{t}\right)$ is the true price. We then take the observed price as $S_{t}^{t}$ obs $=B_{t} q_{t}+A_{t}\left(1-q_{t}\right)$, where $q_{t}=$ Bernoulli[1/2]. Hence the observed price fluctuates randomly between the bid and the ask. We take $S_{0}=\$ 25$, ticksize $=\$ 1 / 16$, and $\sigma_{u}=0.0011$, which implies a fixed daily return volatility of 1.87 percent and an annualized return volatility of thirty percent, assuming 250 trading days per year. For each day's data we calculate both realized and range-based volatility estimates (see text for details), based upon both true and observed returns, using 5-minute, 20-minute and 80- minute underlying price observations. We repeat this 100,000 times, and we show kernel estimates of the corresponding sampling densities below. All volatilities and related summary statistics are expressed in percent.
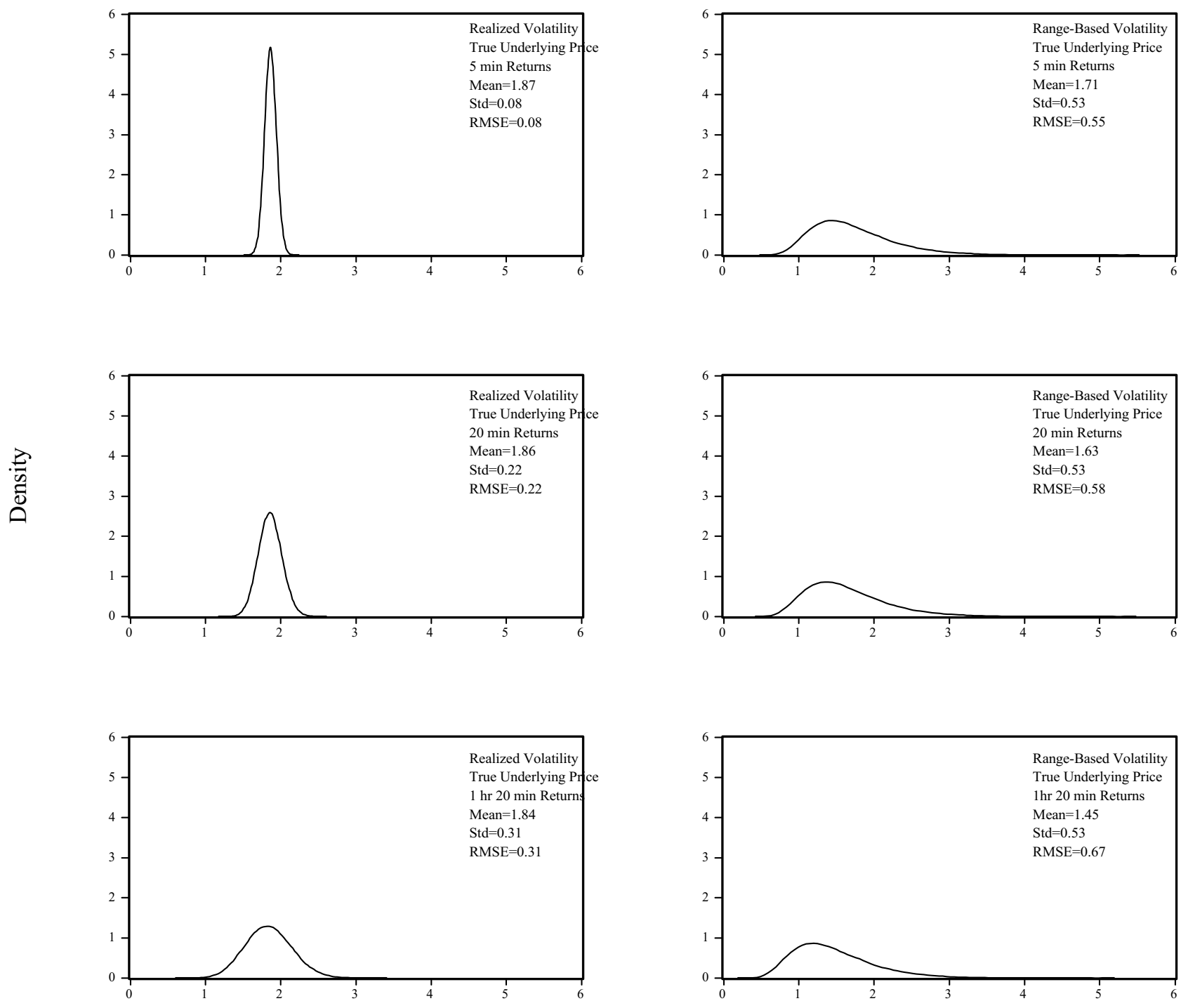

Volatility (Realized or Range-Based, percent) 
Figure 5

\section{Distributions of Realized Volatility and Range-Based Volatility Based on Observed Prices}

We simulate one day of five-minute log prices (289 observations) from the Gaussian logarithmic random walk, $s_{t}=s_{t-1}+u_{t}$, with $u_{t} \sim \mathrm{NID}\left[0, \sigma_{u}^{2}\right]$. Let the bid price be $B_{t}=$ floor $\left[S_{t}-\right.$ ticksize $]$, and let the ask price be $A_{t}=$ ceiling $\left[S_{t}+\right.$ ticksize $]$, where $S_{t}=\exp \left(s_{t}\right)$ is the true price. We then take the observed price as $S_{t}^{o b s}=B_{t} q_{t}+A_{t}\left(1-q_{t}\right)$, where $q_{t}=$ Bernoulli[1/2]. Hence the observed price fluctuates randomly between the bid and the ask. We take $S_{0}=\$ 25$, ticksize $=\$ 1 / 16$, and $\sigma_{u}=0.0011$, which implies a fixed daily return volatility of 1.87 percent and an annualized return volatility of thirty percent, assuming 250 trading days per year. For each day's data we calculate both realized and range-based volatility estimates (see text for details), based upon both true and observed returns, using 5-minute, 20-minute and 80- minute underlying price observations. We repeat this 100,000 times, and we show kernel estimates of the corresponding sampling densities below. All volatilities and related summary statistics are expressed in percent.
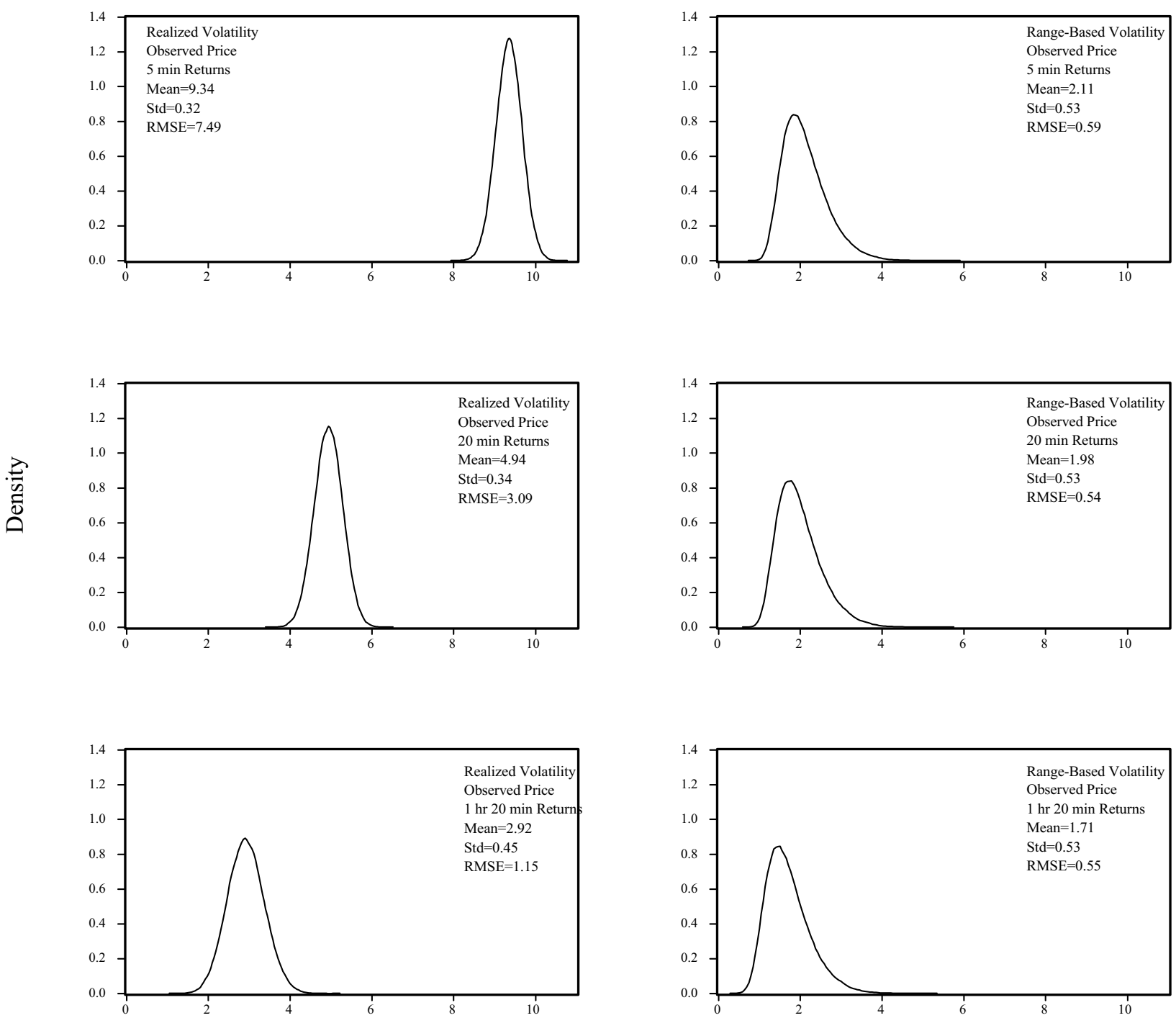

Volatility (Realized or Range-Based, percent) 
Figure 6

Distributions of the Measurement Equation Residuals for

Two-Factor Stochastic Volatility Models for Five Dollar Exchange Rates

We show histograms of the measurement equation residuals for stochastic volatility models estimated using either log absolute returns or the log range as volatility proxy with the best-fitting normal imposed for visual reference, and the corresponding QQ plot, which is a graph of the quantiles of the standardized residual distribution against the corresponding quantiles of a N[0,1] distribution. If the residual is normally distributed, its Gaussian QQ plot is a straight line with a unit slope. The rows correspond to the five currencies examined: the British pound, Canadian dollar, Deutsche Mark, Japanese yen, and Swiss franc.

Log Absolute Returns Residuals

Histogram
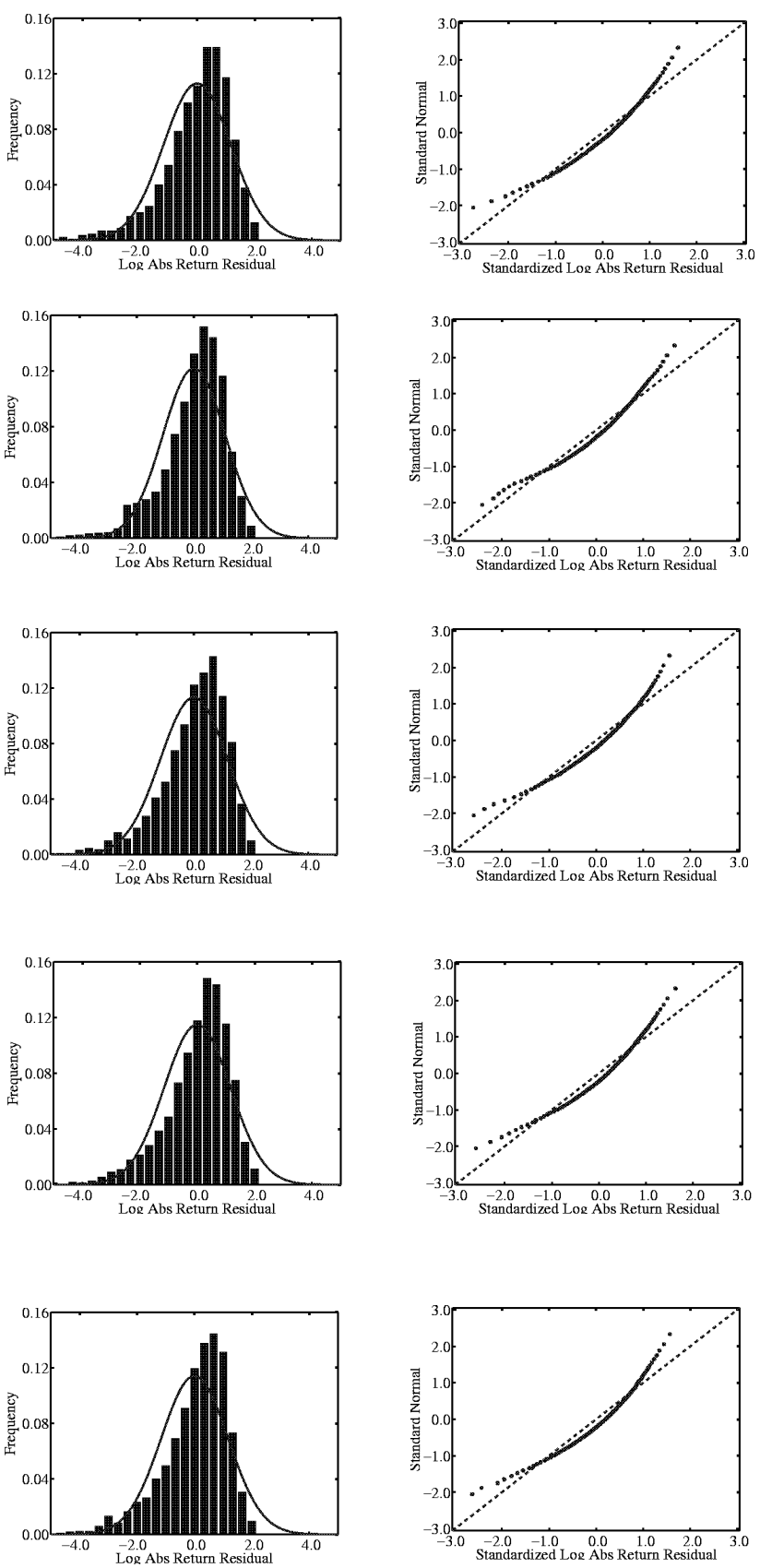

Log Range Residuals Histogram
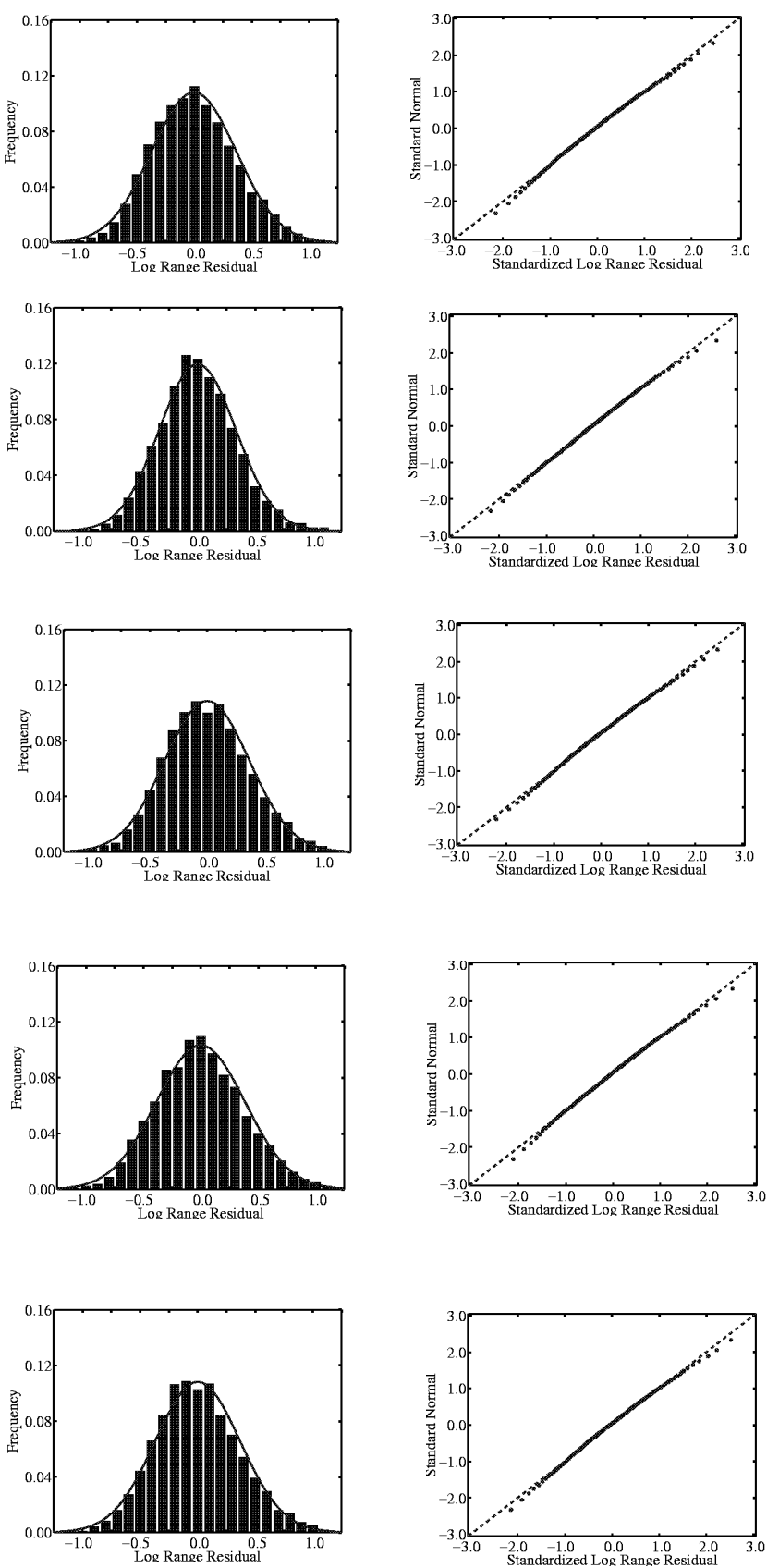